Elsevier Editorial System(tm) for

Nanomedicine: Nanotechnology, Biology, and Medicine

Manuscript Draft

Manuscript Number: JN2016319R2

Title: Micro Patterning of Nanocomposite Polymer Scaffolds Using Sacrificial Phosphate Glass Fibres for Tendon Tissue Engineering Applications

Article Type: Original Article

Keywords: Nanocomposite polymer, phosphate glass fibers, tendon graft, micro pattern scaffolds.

Corresponding Author: Dr. Deepak M Kalaskar, PhD

Corresponding Author's Institution: University college London

First Author: Feras Alshomer, MBBS

Order of Authors: Feras Alshomer, MBBS; Camilo Chaves, MBBS, MSC;

Tiziano Serra, PhD; Ifty Ahmed, PhD; Deepak M Kalaskar, PhD

Abstract: This study presents a simple and reproducible method of micro patterning the novel nanocomposite polymer (POSS-PCU) using a sacrificial phosphate glass fiber template for tendon tissue engineering applications. The diameters of the patterned scaffolds produced were dependent on the diameter of the glass fibers $(15 \mu \mathrm{m})$ used. Scaffolds were tested for their physical properties and reproducibility using various microscopy techniques. For the first time, we show that POSS-PCU supports growth of human tenocytes cells. Furthermore, we show that cellular alignment, their biological function and expression of various tendon related proteins such as scleraxis, collagen I and III, tenascin-C are significantly elevated on the micro patterned polymer surfaces compared to flat samples.

This study demonstrated a simple, reproducible method of micro patterning POSS-PCU nanocomposite polymer for novel tendon repair applications, which when provided with physical cues could help mimic the microenvironment of tenocytes cells. 
UCL Division of Surgery \& Interventional Science, Faculty of Medical Sciences.

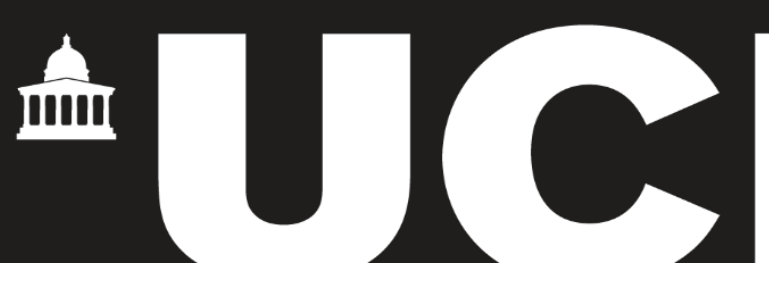

Dear Editor in Chief,

Development of Micro Patterned Scaffolds Using Sacrificial Glass Fibres for Tendon Tissue Engineering Applications

Feras Alshomer, Camilo Chaves, Tiziano Serra, Ifty Ahmed, Deepak M. Kalaskar

We would like to thanks the editor and reviewers for their feedback and comments to improve our work. We have now address all the comments and amended our manuscript.

All changes are highlighted in revised manuscript for you to track easily.

Yours sincerely

Dr Deepak M kalaskar

Lecturer in Nanotechnology and Cellular Engineering

Centre for Nanotechnology and Regenerative Medicine, University College London, London, UK

E-mail: d.kalaskar@ucl.ac.uk 


\section{Reviewer \#4:}

- In this manuscript, the authors have prepared POSS-PCU patterned scaffolds through dissolving the phosphate glass fiber template in water for tendon tissue engineering application. The results demonstrated that the patterned scaffolds could be generated easily and reproducibly. Most importantly, compared to flat film, the patterned scaffolds significantly induced the human tenocytes cells alignment and enhanced the expression of scleraxis, collagen I and III, tenascin-C. This platform may provide a potential strategy for tendon repairmen in the future. Thus, I recommend to accept this manuscript with minor revisions.

\section{Reply:}

We would like to thank the reviewer for his/her comments and constructive feedback to improve our work.

- The authors used $20 \%$ POSS-PCU for flat samples preparation, while $15 \%$ and $5 \%$ POSS-PCU were used for patterned samples fabrication. Justifications are needed to explain why different concentrations of POSS-PCU were chosen and whether the concentration would affect the cell growth.

\section{Reply:}

We thanks reviewer for pointing out this important point. 20\% POSS-PCU solution has higher viscosity. When fibers are put at the interface they submerse into the solution without providing required topographical features. We experimented with various polymer concentrations and finally optimize two stage process, in the first stage $15 \%$ solution was casted and allowed to cure before adding second layer of 5\% solution. Glass fibers are then sandwiched between these two layers, which allowed fibers to be retained at polymer interface. Once completely cured, fibers can be dissolved leaving topographical patterns as shown in results section.

We have now elaborated this point in the methodology under section "2.2 Polymer synthesis and scaffold fabrication".

- Page 15, the authors mentioned that Fig. 5C showed the results of cells cultured in both flat film and patterned scaffolds after 3 weeks in the discussion section, however, it showed "Cell attachment after 24 hrs" in the caption. Please double check the incubation time.

\section{Reply:}

We thanks reviewer for pointing out this mistake. We have now corrected text to make it clear. Fig. 5 indeed corresponds to cell alignment 24-hrs after culture. Text in the manuscript was made clearer as follow: "In order to investigate the potential of micro patterned POSS-PCU as tendon grafts, In-vitro assessment of human 
tenocytes cultured on these samples was done over a period of 3 weeks. The initial assessment after 24-hrs of cell seeding showed that cells attached at similar levels for both flat and micro patterned POSS-PCU (Figure 5 C). However, major differences were observed for cellular alignments 24-hrs after culture, where cells grown on the flat surface failed to orient in a specific direction, whereas on the micro patterned samples, cells aligned in the direction of the grooves (Figure 5 A and B)"

- In addition, Fig 4B indicated that lower cell growths in the patterned scaffolds than the flat samples, which was inconsistent with the results in Fig 5C. Please double check.

\section{Reply:}

We thanks reviewer for their comment, we have tried to clarify this point further.

Figure 4B shows cell growth over long term culture on both on flat and pattern samples, where cell attachment and growth was non-significant between two surfaces at $24 \mathrm{hr}$. This was consistent with Figure 5C where cell attachment is shown at $24 \mathrm{hr}$ post cell seeding. However, when cells are grown on these samples for long term (up to 21days), differences in cell growth was observed as shown in Fig 4C.

We have modified text in section "3.3. Metabolic activity and cellular growth and 3.4. Directing cellular alignment" to clarify this point further.

\section{- Figure $2 \mathrm{~A}$ and $2 \mathrm{~B}$ are in wrong orders.}

\section{Reply:}

We thanks reviewer for pointing this mistake, we have now corrected this in figures section.

- Figure $2 \mathrm{~A}$, why the $\mathrm{pH}$ of deionized water was so low ( 5.7)?

\section{Reply:}

In this experiment, we have used an in-house water purifier to obtain the de-ionized water. Initial assessment point "Day 0" in Figure 2. A showed that level that was having a mean value of 6.1.

The $\mathrm{pH}$ of deionized water is depends on various factors such as its ionic strength and dissolved gases such as carbon dioxide (which is present in the atmosphere) that can decrease $\mathrm{pH}$ value. To avoid this misunderstanding we could have use molecular graded deionized water but this product is relatively expensive and not used in routine. To keep consistent with our experiments, we used available di-ionized water, however reported exact $\mathrm{pH}$ at the time of the measurement.

- Figure 2 B, change "gm" to "mg".

\section{Reply:}


This point is now been addressed in Fig 2.

- Please add unit in the $y$ axis of Figure 3B.

Reply:

This unit is now added to the $\mathrm{Y}$ axis of Figure 3B.

- "deionised" changes to "deionized".

Reply:

The spelling has been modified throughout the manuscript.

Reviewer \#5:

- The authors have presented an interesting work on micro-patterning of a biocompatible and nonbiodegradable POSS-PCU nanocomposite polymer. The authors developed a simple method of micro patterning the POSS-PCU using sacrificial phosphate glass fiber template for tendon tissue engineering applications. The results suggested its suitability for tendon tissue engineering. Furthermore, the authors addressed all the comments one by one. They also correct spelling and grammatical errors throughout the manuscript.

\section{Reply:}

We would like to thank the reviewer for his/her comments and suggestions to improve our work further. 
This study for first time show potential of POSS-PCU nanocomposite for tendon repair applications. Micro-patterning of the POSS-PCU is achieved by using sacrificial phosphate glass fibre template. This micro pattern polymer significantly enhances cellular alignment and biological function of tenocytes compared to unpattern surface, thus bio-mimicking tenocytes microenvironment. This study provides simple, effective and reproducible method of micro patterning polymers for tendon repair applications. 


\section{Flat surface}
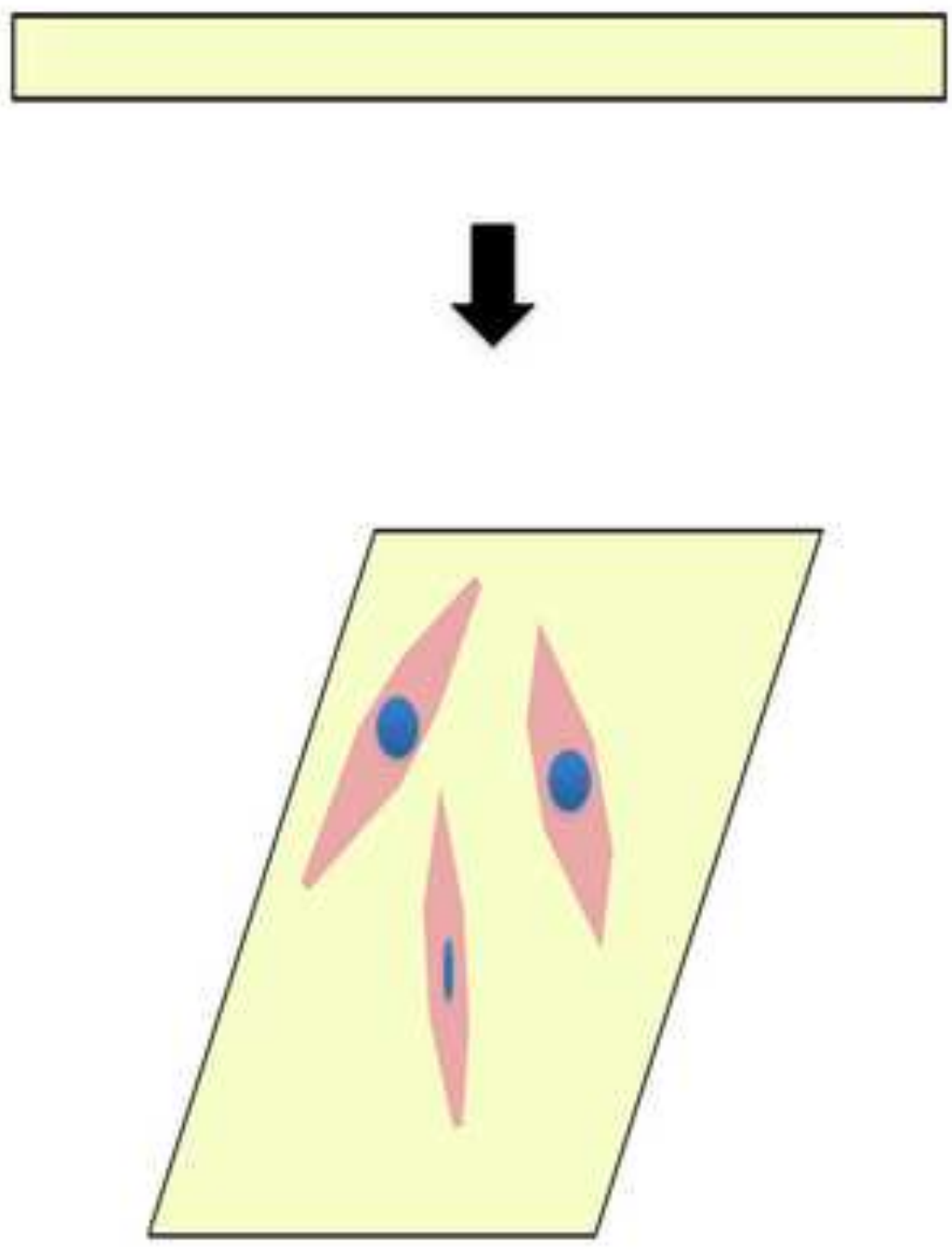

Micro Patterned surface

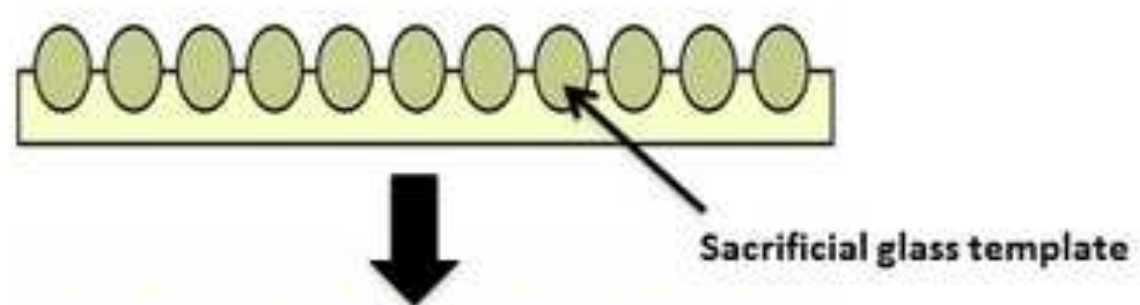

urrurgrar
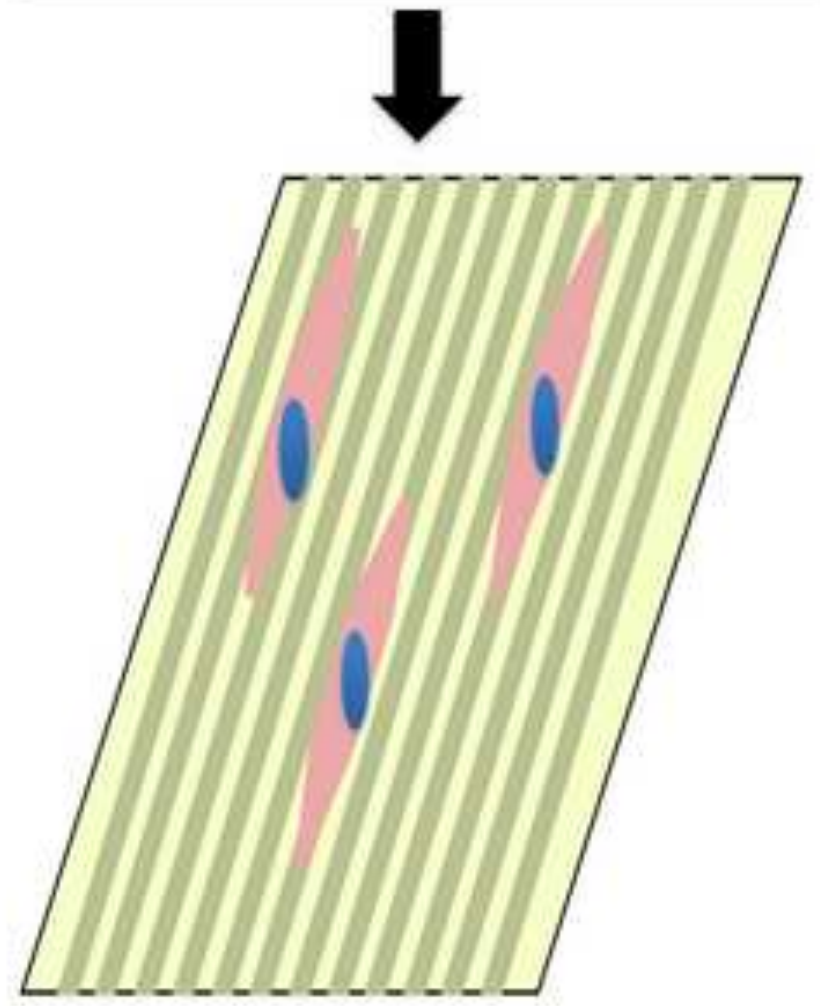


\title{
Micro Patterning of Nanocomposite Polymer Scaffolds Using Sacrificial Phosphate Glass Fibres for Tendon Tissue Engineering Applications
}

\author{
Feras Alshomer ${ }^{1}$, Camilo Chaves ${ }^{1,2}$, Tiziano Serra ${ }^{1}$, Ifty Ahmed ${ }^{3}$, Deepak M. Kalaskar *1 \\ ${ }^{1}$ UCL Centre for Nanotechnology and Regenerative Medicine, Division of Surgery \& Interventional \\ Science, University College London, London, NW3 2PF, United Kingdom. \\ ${ }^{2}$ Université Paris Sud, Orthopedic Surgery, 184, rue du Faubourg-Saint-Antoine, Paris, FR 75012. \\ ${ }^{3}$ Advanced Material Research Group, Faculty of Engineering, University of Nottingham, Nottingham. \\ NG7 2RD, United Kingdom.
}

\author{
*Corresponding author: \\ Dr Deepak M Kalaskar \\ Lecturer in Nanotechnology and Cellular Engineering \\ Centre for Nanotechnology and Regenerative Medicine \\ Division of Surgery \& Interventional Science \\ Rowland Street, NW3 2PF \\ University College London, \\ London, United Kingdom. \\ E-mail: d.kalaskar@ucl.ac.uk
}

Financial Disclosure: None of the authors have any commercial associations or financial relationships that would create a conflict of interest with the work presented in this article.

Funding: This study was funded by Royal Free Charity (Award Number: 167275).

Word count for abstract: 141, Word count for manuscript: 4863, Number of References: 45, Number of figures: 8, Number of tables: 0, Number of Supplementary Online-only files, if any: 1

Keywords: Nanocomposite polymer, phosphate glass fibers, tendon graft, micro pattern scaffolds. 


\section{ABSTRACT:}

This study presents a simple and reproducible method of micro patterning the novel nanocomposite polymer (POSS-PCU) using a sacrificial phosphate glass fiber template for tendon tissue engineering applications. The diameters of the patterned scaffolds produced were dependent on the diameter of the glass fibers $(15 \mu \mathrm{m})$ used. Scaffolds were tested for their physical properties and reproducibility using various microscopy techniques. For the first time, we show that POSS-PCU supports growth of human tenocytes cells. Furthermore, we show that cellular alignment, their biological function and expression of various tendon related proteins such as scleraxis, collagen I and III, tenascin-C are significantly elevated on the micro patterned polymer surfaces compared to flat samples.

This study demonstrated a simple, reproducible method of micro patterning POSS-PCU nanocomposite polymer for novel tendon repair applications, which when provided with physical cues could help mimic the microenvironment of tenocytes cells. 


\section{INTRODUCTION:}

Tendon injuries represent a huge economical burden with an estimated cost of $\$ 30$ billion annually affecting over 110 million patients in USA alone. Repairing these injuries remains an ongoing challenge, with long term impairments and squeal like tendinitis re-rupture and pain $^{1}$. Surgical options are considered when conservative treatment fails to either repair or restore tendon function. Tendon grafts are commonly harvested from the same patient to replace diseased tendon, however this leads to further injury and defects ${ }^{2}$. Allografts and synthetic tendon grafts are also used, however they are limited in use due to possible immunological rejection and poor tissue integration, respectively ${ }^{3-5}$.

Cells respond to their microenvironment, which is a complex mixture of various physical, chemical and biological cues ${ }^{6,7}$. Development of appropriate biomaterial strategies to repair specific tissue types should consider incorporation of these cues during their fabrication. Tendon tissue is made up of a hierarchal structure of collagen fibers, which are aligned, in parallel fashion. Tenocytes naturally follow these fibrous topographical features within their structure. In order to bio mimic tendon structure, one strategy is the incorporation of micro or nanoscale topography that mimics the native collagen fiber arrangement. Previous studies showed elevated levels of tenomodulin by tenocytes when grown on micro-grooved silicon membranes ${ }^{8}$. When mesenchymal cells were grown over aligned collagen threads they showed increased expression of scleraxis and tenomodulin ${ }^{9,10}$. Additionally, when aligned PLGA nanofibers were used as a scaffold for tendon regeneration, increased alignment, proliferation, and matrix deposition ${ }^{11}$ together with increased tenogenic differentiation was observed with Tendon derived stem cells (TDSCs) ${ }^{12}$.

In this study, we used a nanocomposite polymer called polyhedral oligomeric silsesquioxanepoly (carbonate-urea) urethane (POSS-PCU) polymer synthesised in our lab. It is composed of a PCU backbone cross linked with POSS nanoparticles. Its specific 
nanostructure enhances the mechanical properties and biocompatibility of POSS and PCU composite. Its biocompatibility is linked to cage-like silsesquioxane molecules containing an inner inorganic framework composed of silicone and oxygen ${ }^{13}$. The improved mechanical properties were related to the incorporation of POSS building blocks into the polyuriaurethane forming a hybrid of inorganic-organic co-polymer improving its eventual elasticity 14-15. The POSS-PCU co-polymer was shown to have amphiphilic properties with hydrophobic tendency from the POSS molecules ${ }^{13}$. POSS-PCU is a non degradable polymer ${ }^{16}$ with no associated toxicity ${ }^{17}$. This material has been investigated thoroughly for different applications including; development of skin ${ }^{18}$, lacrimal ducts ${ }^{19}$, tissue engineered larynx ${ }^{20}$, vascular grafts ${ }^{21}$ and tissue engineered auricle ${ }^{22}$ among others.

In this work, fabrication of the surface micro topography on this polymer was achieved using fully soluble phosphate glass fibers (of formulation $50 \mathrm{P}_{2} \mathrm{O}_{5}-30 \mathrm{CaO}-20 \mathrm{Na}_{2} \mathrm{O}$ ) ${ }^{23-25}$ as a sacrificial template for the first time. The glass fibers were incorporated within the POSSPCU scaffold during fabrication and later removed by dissolving in water to create micro patterned scaffolds. These scaffolds were then tested using human tenocytes to evaluate their potential for development of artificial tendon grafts. This study aims to provide simple and cost effective surface patterning technique for POSS-PCU nanocomposite polymer. Fabrication technology can be translated to various other biocompatible polymers for similar applications.

\section{MATERIALS AND METHODS}

\subsection{Materials}

Fully soluble phosphate glass fibers with ternary composition of $50 \mathrm{P}_{2} \mathrm{O}_{5}-30 \mathrm{CaO}-20 \mathrm{Na}_{2} \mathrm{O}$ were prepared using the following precursors, $\mathrm{NaH}_{2} \mathrm{PO}_{4}, \mathrm{CaHPO}_{4}$ and $\mathrm{P}_{2} \mathrm{O}_{5}$ (Sigma Aldrich, UK). The precursors were dried at $350^{\circ} \mathrm{C}$ for 30 mins, followed by melting at $1100^{\circ} \mathrm{C}$ for 90 
mins. The molten glass was then poured onto steel plate and left to cool to room temperature. The glass frit obtained was then used to manufacture phosphate-glass fibers using a specially designed in house melt drawn fiber production facility (Faculty of Engineering, University of Nottingham, U.K.) ${ }^{23-25}$.

\subsection{Polymer synthesis and scaffold fabrication}

The process of manufacturing of Polyhedral Oligomeric Silsesquioxane Poly (Carbonateurea) Urethane (POSS-PCU) has been described previously in details ${ }^{16,26}$.

Two types of POSS-PCU samples were fabricated, flat and patterned by casting polymer solution into a glass mold. The flat sample was simply produced by casting $20 \%$ of POSSPCU polymer solution into the glass mold $(16 \times 16 \mathrm{~cm})$. The sample was then placed in an oven at $65^{\circ} \mathrm{C}$ for 16 hours until solvent had evaporated (Figure $1 \mathbf{A}$ ). In order to produce pattern surfaces, we use soluble glass fibers of know diameter. When fibers are put at the interface of liquid polymer film, they submerse into the solution without providing required topographical features. We experimented with various polymer concentrations and finally optimize two stage process, in the first stage $15 \%$ solution is casted and allowed to cure for 16 hours. In second stage, densely packed Phosphate glass fiber mesh was then laid on top of the casted layer. Finally, 5\% POSS-PCU solution was poured onto the uniformly distributed fibers so that the fibers were sandwiched between POSS-PCU polymer films (as shown in Figure $1 \mathrm{~B})$. Samples were then cured in oven at $65^{\circ} \mathrm{C}$ for 16 hours. Two stage process allowed fibers to be retained at the interface of the casted films, which otherwise sink into the polymer without providing topographical interface intended in this study.

Scaffold films were then cut into circles of $1.6 \mathrm{~cm}$ diameter were used in the assessment process. In order to produce patterned surfaces, samples were kept in deionized water until the glass fiber had completely dissolved using constant agitation on a mechanical roller, followed daily with water exchange. Dissolution of fibers was monitored on a regular basis by checking the dry weight of the samples along with the $\mathrm{pH}$ of the water every day. Resultant scaffolds both flat and patterned were stored in a desiccator until further use. 


\subsection{PHYSIO-CHEMICAL CHARACTERISARTION}

\subsubsection{Confirmation of dissolution of degradable glass fibres}

To ensure complete removal of the biodegradable glass fibers, both changes in $\mathrm{pH}$ and weight of samples were monitored over a period of 7 days. Briefly, scaffold samples were initially weighed then incubated with fixed volume of deionized water with a mechanical roller at room temperature. Initial water's $\mathrm{pH}$ was recorded prior to the incubation as a reference point. Change in $\mathrm{pH}$ was measured every day using $\mathrm{pH} 2100$ Bench top meter (OAKTON Instruments Ltd. USA) followed by once daily water change. Additionally, samples were air dried and weighed to assess resultant change in weight after dissolution of fibers as a complimentary method. Readings from 4 different samples were obtained at each assessment point $(n=4)$.

\subsubsection{Surface topography analysis using Scanning Electron Microscope (SEM)}

Morphology of the polymer films both flat and patterned were analyzed using SEM. Samples were mounted on aluminum stubs using carbon tap, and coated with gold (SC500 EM Scope). Samples were analyzed using SEM SIGMA Series of Field Emission Scanning Electron Microscope, LS15 EVO HD (Carl Zeiss, USA).

\subsubsection{Reproducibility of patterned scaffolds}

Reproducibility of patterned scaffolds was assessed using light microscopy. Samples were placed on glass slides for light microscopic imaging. Nikon TMS-F light microscope with (INFINITY CAPTURE software) was used to obtain three random images per sample from three scaffold samples from three separate production patches $(n=3)$. Spacing between patterns formed after dissolution of glass fibers were analyzed using ImageJ analysis software. Areas with spacing visualized indicated light passing through the sample's gaps.

\subsubsection{Water contact angle (WCA)}


Wettability of the scaffolds due to changes in topography was documented using WCA measurements. Briefly, static water contact angle was measured on samples using KRÜSS's DSA 100 water contact angle analysis machine (KRÜSS, Germany). WCA was measured for both flat and patterned samples $(n=5)$ with 3 random readings per sample. Briefly, 3- $\mu 1$ deionized water was dispensed through a needle. Images of WCA were captured using high speed camera and analysis was conducted using KRÜSS drop shape analysis software.

\subsection{IN-VITRO CELLULAR ASSESSMENT}

\subsubsection{Cell culture and seeding.}

Human Tenocyte harvested from a 61 year old male patient were purchased from Zen Bio, USA, and cultured as per manufacturer's instructions. Briefly, the cells were cultured in $75 \mathrm{~cm}^{2}$ tissue culture flask coated with collagen at $37^{\circ} \mathrm{C}$ and $5 \% \mathrm{CO}_{2}$ in high glucose DMEM (Gibco®, Life Technologies, UK), along with $1 \%$ penicillin/streptomycin and $10 \%$ Foetal bovine serum (FBS), media was changed every 2-3 days. Cells were passaged when reached $80 \%$ confluency. For in-vitro testing, polymeric scaffolds were sterilized using $70 \%$ ethanol for 30 min prior to cell seeding and washed 3 times with PBS. Cells were seeded at a density of 20,000 cells $/ \mathrm{cm}^{2}$. Circular scaffolds of $1.6 \mathrm{~cm}$ diameter were used that fits into 24-well plates. Four repeats for each of flat and patterned scaffold groups were utilized $(n=4)$. Glass cover slips were used as control.

\subsubsection{Quantification of Cellular alignment}

Cellular alignment on flat and patterned surfaces was quantified using actin cytoskeleton staining after 24 hours of cell seeding. Detailed procedure is outlined in supplementary information. Images were captured randomly from 3 different areas per sample in triplicates $(n=4)$. For each image, an ellipse was fitted to the cell outline and the major axis was considered as the direction of angle orientation. For the control group (glass coverslip) and 
flat samples, the angle between major ellipse axis and image $\mathrm{x}$-axis was calculated. For the study group, both the angle of the ellipse major axis and the angle along the grooves direction were recorded. Accurate angulation values were obtained by subtracting the major axis calculated from the reference $\mathrm{x}$-axis or grooves and denoted as $(\boldsymbol{\alpha})$. Values that were close to zero degree were considered in alignment with the reference geometry whereas values close to 90 degrees were perpendicular to the reference points ${ }^{27,28}$.

\subsubsection{Cellular metabolic activity and growth.}

Cellular metabolic activity and growth were assessed using Alamar blue and DNA assay respectively. Cellular metabolic activity was monitored using Alamar blue dye reduction, analysis was carried out as per manufacturer's instructions (Invitrogen, UK). Briefly, samples were incubated with $10 \%$ Alamar blue dye in DMEM for 4 hours at $37^{\circ} \mathrm{C}$. $200 \mu \mathrm{l}$ of reduce dye was transferred to 96 well plate and resultant fluorescent readings were obtained using Fluoroskan ascent FL reader (Biochrome Ltd, UK) under 530nm excitation and 620nm emission. The metabolic activity of human tenocytes at passage 4 was assessed over a period of 21 days. Additionally, cellular growth was assessed using bisBenzimide Hoechst 33258 assay (DNA assay) (Sigma-Aldrich, UK). A series of freeze thawing cycles were used to lyse the cells followed by mixing with Hoechst-33258 dye solution. Sample Fluorescence was then measured using Fluoroskan reader having set at 360nm excitation and 460nm emission. Standard curve was constructed as described in the supplier's kit followed by DNA quantification. Total of 4 replicates were used during analysis for each test condition $(n=4)$.

\subsubsection{Immunostaining of tendon specific Extracellular matrix proteins}

Expressions of different tendon related ECM proteins (collagen I, collagen III, tenascin-C, and scleraxis) were evaluated at the end of the assessment duration (day 21). Samples were fixed with $4 \%(\mathrm{w} / \mathrm{v} \%)$ Paraformaldehyde for 20 minutes at room temperature. This was followed by permeabilization for 5 min using $0.1 \%$ (v/v \%) Triton X-100 in PBS. Samples 
were then blocked using 1\% (w/v \%) bovine serum albumin (all from Sigma-Aldrich, UK) in PBS for 30 minutes. Primary antibodies diluted in 1\% BSA were incubated overnight. Samples were rinsed with $0.1 \%$ Tween in PBS. Fluorescent secondary antibodies diluted in $1 \%$ BSA were then incubated with their corresponding targeted primary antibodies for 1 hour at room temperature. Samples were washed three times with $0.1 \%$ tween in PBS and stained with DAPI for 4 minutes, which were then mounted using fluoromount aqueous mounting media (Sigma-Aldrich, UK) and imaged using Leica DMI 4000b Fluorescence microscope.

\subsubsection{Collagen quantification.}

The total collagen production on the scaffolds was quantified using $\mathrm{SirCol}^{\mathrm{TM}}$ collagen dye binding assay kit, (Biocolor Ltd., UK) as per manufactures instructions. Details of exact procedure is provided in supplementary information. Non-seeded scaffolds and glass cover slip were used as control. Assessment of collagen production was done at $7^{\text {th }}, 14^{\text {th }}$, and $21^{\text {st }}$ days. At each assessment point four samples for each of the studied scaffold and control groups were assessed $(n=4)$.

\subsection{STATISTICAL ANALYSIS.}

All error bars on data are expressed as standard error of mean (SEM). The statistical significance was determined at 95\% level using a one-way ANOVA, where * represents $\mathrm{p}<$ $0.05, * *$ represents $\mathrm{p}<0.01$ and $* * *$ represent $\mathrm{p}<0.001$. Post-hoc statistical analysis of the means of individual groups was performed using Tukey's test.

\section{RESULTS}

\subsection{Fabrication of micro topographical patterned surfaces using sacrificial glass fibers}

Surface topography was produced by dissolving embedded phosphate glass fibers from the scaffold using deionized water over a period of 7 days. Measuring the dry weight of the sample with embedded fibers over a period of 7 days confirmed fiber dissolution (Figure 2 
A). From the graph it was clear that the weight of the sample decreased over the 7-day period of the study, suggesting no more leaching of glass fibers from the scaffold. The $\mathrm{pH}$ of the media was also monitored to indirectly check for the removal of the glass fibers (Figure 2. B). The $\mathrm{pH}$ decreased at day 1 and then increased to the $\mathrm{pH}$ of deionized water after 1 day on incubation. This confirmation along with weight loss results suggested that the glass fibers had degraded from the POSS-PCU samples.

Scanning electron microscopy further confirmed removal of the glass fibers and production of micro topography at the interface of the POSS-PCU samples (Figure $2 \mathbf{C}$ and D). It was clear from the Figure that fibers were embedded at the scaffold interface prior to fiber removal. However after incubation with deionized water over 1 day, dissolution of the fibers produced micro topographical features resembling the fibers, which have been labelled as "patterned" scaffold. Analysis of the pattern using image analysis $(n=4)$, confirmed the average micro topographic width of $15 \pm 1.2 \mu \mathrm{m}$, which was similar to the diameter of the glass fibres used.

Reproducibility of the micro topography during different batches of samples was assessed using light microscopy imaging as reported in the materials and method section. Assessment of three different batches showed that the gap between the micro patterns was $86 \pm 6 \mu \mathrm{m}$ (Figure 3 A.). No significant difference was found between the 3 production batches $(p>0.05)$, confirming reproducibility of the fabrication process.

\subsection{Surface wettability}

Water contact angle (WCA) measurements of both flat and pattern POSS-PCU samples (Figure 3 B) showed changes in wettability of the samples where the POSS-PCU was inherently hydrophobic with an average WCA of $98 \pm 2^{\circ}$. However, after POSS-PCU was patterned to produce micro topography using aligned fibers, the WCA increased to $111 \pm 2^{\circ}$ ( $p$ $\leq 0.01)$ 


\subsection{Metabolic activity and cellular growth.}

Metabolic activity and cell growth of the Tenocytes was monitored using AlamarBlue ${ }^{\circledR}$ and DNA assay respectively as shown in Figure 4.

Metabolic activity of the cells on both flat and patterned POSS-PCU samples showed similar behavior until 14 days, however after 21 days significant increase $(\mathrm{p}<0.05)$ in metabolic activity was observed on the patterned samples as compared to the flat POSS-PCU samples. On the other hand, DNA assay showed increase in cell numbers until 7 days on flat samples, after which DNA content reached saturation, suggesting that cells had reached confluence. However, the cells maintained their viability and remained attached to the flat surface post 21 days. On the micro patterned surfaces however, cell growth showed no further increase in cell growth post 7 days and remained stable until last assessment point of 21 days.

\subsection{Directing cellular alignment}

After day 1 of cell culture, tenocytes seeded on the samples displayed self organized behavior. Cells seeded on flat POSS-PCU samples did not exhibit any tendency of alignment due to the absence of directional cues. However, on the micro patterned samples cells were oriented along the direction of the micro patterns as shown in Figure 5 A. Quantification of cellular alignment showed average angle of $48 \pm 3^{\circ}$ for cells grown on flat surface, compared to $2 \pm 0.5^{\circ}$ for cells grown on micro patterned samples with significant difference $(p<0.001)$ (Figure 5 B.). The angle of cytoskeleton alignment of major axis of cells of less than $10^{\circ}$ was considered to be well aligned. Results from this study thus confirmed that cells were aligned in response to the micro pattern created. The initial assessment after 24-hrs of cell seeding showed that cells attached at similar levels for both flat and micro patterned POSS-PCU (Figure 5 C).

\subsection{Total Collagen production}


Functional activity of seeded Tenocytes on different scaffolds was assessed through total collagen production as shown in Figure 6. Collagen production increased significantly $(p<0.001)$ on the micro patterned samples after day 14 and maintained collagen level until 21 days of analysis, compared to flat-POSS-PCU samples.

\subsection{Expression of Tendon Specific ECM proteins}

Immunofluorescent staining was performed to determine cellular distribution and tendon related ECM proteins. Expression of collagen-I, collagen -III, tenascin-C, and scleraxis were assessed 21 days post seeding as shown in Figure 7 and 8.

Cells expressed all markers on both flat and patterned POSS-PCU samples. Differences were clearly visible in orientation of cells and expression of ECM proteins between the flat and micro patterned samples. On the micro patterned samples, the cells had deposited tenocyte associated proteins along the micro patterns, whereas on the flat samples ECM proteins were randomly distributed. Cells seemed to express higher level of collagen -III, tenascin-C and scleraxis, however immune fluorescence is a qualitative technique and thus further quantification of individual proteins will need to be considered in future analyses.

\section{DISCUSSION}

Scaffolds which can mimic the native topographic matrix features have proven to be important in the field of Tissue Engineering (TE). Topographical cues can provide cells with directional alignment, which can help certain type of cells mimic their native environment ${ }^{6}$. The concept of physical guidance (stereotropism) was established after the initial work of Harrison in 1911, who directed cell growth along spider web fibers ${ }^{7}$. This work later evolved with advancements in different fabrication techniques for various materials including collagen, polystyrene, silicon and metals creating topographically smart surfaces with an essential impact on cell adhesion, morphology, subsequent spreading and differentiation ${ }^{29-33}$. 
The use of fully soluble phosphate glass fiber as a scaffold fabrication tool was initially investigated by Nazhat et al. ${ }^{25}$. In their study, phosphate glass fibers were used to create micro-channels within plastic compressed collagen gel aiming to improve cell perfusion, nutrient delivery and potential vascularization throughout the dense construct.

In this study, the use of soluble phosphate glass fibers with ternary composition of $50 \mathrm{P}_{2} \mathrm{O}_{5}-$ $30 \mathrm{CaO}-20 \mathrm{Na}_{2} \mathrm{O}$ as temporary components to indirectly introduce micro patterned features at the polymer interface were investigated. Dimensions of micro topographical features depended on the size of the glass fibers used. Since these fibers are fully soluble, they were used as a sacrificial template to introduce the micro topographical patterns on POSS-PCU polymer. In this study the main aim was to investigate whether this novel polymer was able to support tenocyte growth and provided with physical cues as to whether it could be used as a potential tendon graft.

Physical properties and degradation behavior of these fibers was previously reported by Ahmed et al. ${ }^{23-25}$. Thus, the main focus in this study was on the physical and biocompatible characterization of the scaffolds produced. The fabrication process showed that the phosphate glass fibers were removed after 1-day incubation in deionized water at room temperature. These results were confirmed by the dry weight measurements and also by monitoring $\mathrm{pH}$ of the water as shown in Figure $2 \mathrm{~A}$ and B. SEM analysis further confirmed complete fiber removal, which yielded the surface micro topography together with hollow tubular structures reflective of the original fiber diameter, orientation, and packing. Image analysis of the patterned features produced revealed the production of micro grooves with an average width that resembled the fiber diameters (Figure $\mathbf{2} \mathbf{C}$ and $\mathbf{D}$ ).

Various methods such as photolithography ${ }^{28}$, thermal pressing micro patterning ${ }^{34}$, the use of electrospun fibers scaffolds ${ }^{35}$ at micro and nano scale level have been investigated by various authors for tendon tissue engineering. Kappor et al. used photolithography to create 50-250 
$\mu \mathrm{m}$ micro topographical ridges on glass substrates. When they seeded tenocytes on samples possessing $50 \mu \mathrm{m}$ features, they showed improved cellular alignment and adhesion ${ }^{28}$. Micro topographical patterns with grooves and stepped features were also created on dried collagen hydrogels with 30,75 and 150 grooves $/ \mathrm{mm}$ of $2 \mu \mathrm{m}$ depth, which also showed to support cellular alignment ${ }^{30}$. Shi $\mathrm{X}$ et al. ${ }^{34}$ reported on the fabrication of micro patterned drug (melatonin) laden poly(lactic-co-glycolic acid) (PLGA) films using indirect Teflon stamps to impose cellular alignment. It was found that seeded fibroblasts exhibited a significant alignment that is parallel to the direction of grooves with greater degree over scaffolds possessing $50 \mu \mathrm{m}$ grooves.

Lu et al. ${ }^{36}$ fabricated capillary channels with open grooves on PLA and PET polymers fibers. The groove depth ranged from $5-15 \mu \mathrm{m}$ and with a width of $10 \mu \mathrm{m}$ in a three dimensional fashion. Again, a significant effect for cell alignment was observed together with the potency of fluid transport across the open channel fibers compared to the round fibers used as control. The outcomes of the results in this study are of particular interest as the fabrication of both micro topographical grooves together with hollow channels at the scaffold interface were achieved. Majority of the previously described fabrication techniques involved the use of substrate molds where micro topographical features were translated into the scaffold material of interest in a controlled fashion. However, in current study, the confounding variable was that topographical features created depended largely on the size and spatial distribution of the degradable phosphate glass fibers used. Reproducibility of this method was confirmed by producing 3 different batches of scaffolds and measuring the average spacing between the micro patterned grooves, which were found to be $86 \pm 6 \mu \mathrm{m}$ with no detectable significant difference among different production batches.

Topographical features resulted in a change in wettability of the samples, where flat POSSPCU samples exhibited average WCA of $98 \pm 2^{\circ}$, and the micro patterned POSS-PCU showed 
higher $(\mathrm{P} \leq 0.01)$ WCA of $111 \pm 2^{\circ}$. These findings seemed to follow Wenzel's theory, according to which hydrophobic surfaces become more hydrophobic with increasing surface roughness ${ }^{37}$.

In order to investigate the potential of micro patterned POSS-PCU as tendon grafts, In-vitro assessment human tenocytes were cultured on these samples over a period of 3 weeks. The initial assessment after 24-hrs of cell seeding showed that cells attached at similar levels for both flat and micro patterned POSS-PCU (Figure 5 C). However, major differences were observed for cellular alignments, where cells grown on the flat surface failed to orient in a specific direction, whereas on the micro patterned samples, cells aligned in the direction of the grooves (Figure 5 A and B). It is well known that cells respond to physical cues, which can affect their morphology and proliferation [39]. To further investigate if the guided alignment of cells could result in favorable cellular function for tendon regeneration, longer term cell studies were undertaken.

Screening of cellular viability and metabolic activity was investigated through AlamarBlue ${ }^{\circledR}$ assay to assess biocompatibility of the scaffold produced (Figure 4 A). A significant $(\mathrm{p}<0.05)$ increase in metabolic activity of cells was observed on the micro patterned samples compared to the flat POSS-PCU samples.

This finding supported previous studies evaluating the effect of cellular alignment on subsequent metabolic activity. Vaquette et al. ${ }^{38}$ showed that bone marrow MSCs when seeded on aligned poly(L-lactic-co-e-caprolactone) electrospun microfiber membranes showed statistically higher metabolic activity compared to random fibers after 14 days of culture.

Interestingly, the metabolic activity of the cells increased on the micro patterned samples compared to flat samples as shown by Alamar blue assay, however, the DNA assay showed lower cell growth on the patterned surfaces compared to the flat samples (Figure 4 B). This 
finding was also of particular interest as previous reports in the literature showed similar patterns also reduced cellular growth in the presence of these topographical features. Wan et al. ${ }^{39}$ showed an improved OCT-1 osteoblast-like cells adhesion on PLA and polystyrene substrates possessing nano, and micro topography, however no enhancement of cell proliferation was seen compared to smooth controls. Bashur et al. ${ }^{40}$ showed similar findings of no significant enhancement of cellular growth (Hoechst 33258) of mesenchymal progenitor cells seeded on aligned electrospun Polyurethane fiber meshes. Furthermore, Kapoor et al. ${ }^{28}$ showed lower tenocytes cell density at confluence (80hrs of seeding) with glass substrates having $50 \mu \mathrm{m}$ grooves compared to other constructs with wider groove widths and unpatterned control surfaces. The steady state of cellular growth and maintenance was linked to the effect of the topographic features introduced ${ }^{41}$.

The importance of guiding cell morphology and alignment was shown to have an effect over subsequent expression of tendon related matrix. Li et al. ${ }^{42}$ showed that elongated human tendon fibroblasts were associated with higher levels of collagen I expression when compared to less stretched cells. The work of Vaquette et al. ${ }^{38}$ also showed increased production of both collagen I and III over aligned microfibers/knitted scaffolds compared to tissue culture plastic controls. Moreover, cell seeding over electrochemically aligned collagen threads showed increased expression of scleraxis and tenomodulin with their essential role in tendon matrix assembly ${ }^{9,10}$. Sahoo et al. also reported increased expression levels of collagen I, collagen III, and tenascin-C over polymeric electrospun nanofiber scaffolds ${ }^{43}$. They suggested that this could be due to the associated changes in focal adhesion orientation, actin cytoskeleton deformation, and the distribution of traction forces that guided cell mechanotransduction.

To investigate if such effect were present on POSS-PCU samples, expression of different tendon related matrix proteins (collagen I, collagen III, tenascin-C, and scleraxis) were 
analyzed using immune staining. Qualitative assessment of immunofluorescent staining showed a strong expression of different tendon related ECM proteins on the micro-patterned and flat samples as in (Figure 7 and 8). Difference in the expression levels of different collagen types produced coincided with previously reported results. Sahoo et al. ${ }^{43}$ showed increased level of collagen III compared to collagen I on cell seeded polymeric electro spun nano fiber scaffolds. This was linked to the fact that collagen III was usually expressed in the early inflammatory and proliferative phase of tendon healing which was later replaced with collagen I in the remodeling phase.

In this study, the effect of topographical modification over total collagen production showed a significant increase in amount of collagen produced on the micro patterned POSS-PCU compared to flat POSS-PCU samples $(\mathrm{P}<0.001)$ (Figure 6). This observation was in line with previous studies where similar results were reported on various aligned fibrous scaffolds 44,45

Introduction of controlled topography enabled tenocyte cells to align along the patterns produced. Achieving this alignment of cells was the intention of this work, as it could potentially assist cells to mimic their native environment. As a consequence of this alignment, we noted that the cells had different metabolic activity and an increase in collagen production was also observed. It is unlikely this effect is caused due to changes in wettability of the surface, as difference water contact angles for Flat $\left(98 \pm 2^{\circ}\right)$ and the patterned POSSPCU $\left(111 \pm 2^{\circ}\right)$, even though significant, it remains quite small. The surface topography however, is likely to be the major governing factor which has a significant effect on cellular activity and ECM production, as highlighted in studies reported before.

Whilst this study demonstrated the potential role to use a polymeric POSS-PCU system as a scaffold in tendon TE, the study was limited by different factors that might be the scope of 
future work. For example, although production of various tendon related ECM proteins was increased over scaffolds possessing micro-topographic features, quantitative analysis and gene profiles of various ECM molecules produced will further help in understanding tissue integration and tendon function. In addition assessment of the mechanical properties of the scaffolds produced together with the effect of dynamic culturing conditions will be further investigated as these would be considered to be important for aimed clinical application.

\section{CONCLUSIONS:}

This study demonstrated a simple and cost effective method of micro patterning polymer surfaces using sacrificial micro phosphate glass fibers. Novel POSS-PCU polymer was shown for the first time to support tenocyte viability, proliferation and functional activity. This was particularly enhanced with the introduction of micro topographical features.

Tenocytes were shown to exhibit native elongated morphology together with higher metabolic activity on micro patterned surfaces compared to flat controls. Additionally, contact guidance of cell alignment over seeded patterned-POSS-PCU was associated with increased collagen production, along with all essential ECM proteins.

These encouraging findings support future investigative work exploring potential applications of the micro patterned polymer surfaces produced as a tendon graft for tendon repair applications.

\section{Acknowledgments}

None 


\section{References}

1. R. James, G. Kesturu, G. Balian and A. B. Chhabra, Tendon: biology, biomechanics, repair, growth factors, and evolving treatment options. The Journal of hand surgery. 2008;33:102-12

2. $\quad$ C.-F. Liu, L. Aschbacher-Smith, N. J. Barthelery, N. Dyment, D. Butler and C. Wylie, What we should know before using tissue engineering techniques to repair injured tendons: a developmental biology perspective. Tissue Engineering Part B: Reviews. 2011;17:165-76

3. K. Tadokoro, N. Matsui, M. Yagi, R. Kuroda, M. Kurosaka and S. Yoshiya, Evaluation of hamstring strength and tendon regrowth after harvesting for anterior cruciate ligament reconstruction. The American journal of sports medicine. 2004;32:1644-50

4. M. Krueger-Franke, C. Siebert and S. Scherzer, Surgical treatment of ruptures of the Achilles tendon: a review of long-term results. British journal of sports medicine. 1995;29:121-5

5. H. K. Uhthoff, G. Trudel and K. Himori, Relevance of pathology and basic research to the surgeon treating rotator cuff disease. Journal of orthopaedic science. 2003;8:449-56

6. R. G. Flemming, C. J. Murphy, G. A. Abrams, S. L. Goodman and P. F. Nealey, Effects of synthetic micro- and nano-structured surfaces on cell behavior. Biomaterials. 1999;20:573-88 7. R. G. Harrison, ON THE STEREOTROPISM OF EMBRYONIC CELLS. Science. 1911;34:279-81

8. J. Zhu, J. Li, B. Wang, W. J. Zhang, G. Zhou, Y. Cao, et al., The regulation of phenotype of cultured tenocytes by microgrooved surface structure. Biomaterials. 2010;31:6952-8

9. V. Kishore, W. Bullock, X. Sun, W. S. Van Dyke and O. Akkus, Tenogenic differentiation of human MSCs induced by the topography of electrochemically aligned collagen threads. Biomaterials. 2012;33:2137-44

10. V. Kishore, J. A. Uquillas, A. Dubikovsky, M. A. Alshehabat, P. W. Snyder, G. J. Breur, et al., In vivo response to electrochemically aligned collagen bioscaffolds. Journal of Biomedical Materials Research Part B: Applied Biomaterials. 2012;100:400-8

11. K. L. Moffat, A. S.-P. Kwei, J. P. Spalazzi, S. B. Doty, W. N. Levine and H. H. Lu, Novel nanofiber-based scaffold for rotator cuff repair and augmentation. Tissue Engineering Part $A$. 2008;15:115-26

12. Z. Yin, X. Chen, J. L. Chen, W. L. Shen, T. M. H. Nguyen, L. Gao, et al., The regulation of tendon stem cell differentiation by the alignment of nanofibers. Biomaterials. 2010;31:2163-75 13. S. B. Rizvi, S. Y. Yang, M. Green, M. Keshtgar and A. M. Seifalian, Novel POSS-PCU Nanocomposite Material as a Biocompatible Coating for Quantum Dots. Bioconjugate chemistry. 2015;26:2384-96

14. R. Y. Kannan, H. J. Salacinski, M. J. Edirisinghe, G. Hamilton and A. M. Seifalian, Polyhedral oligomeric silsequioxane-polyurethane nanocomposite microvessels for an artificial capillary bed. Biomaterials. 2006;27:4618-26

15. H. Ghanbari, B. G. Cousins and A. M. Seifalian, A nanocage for nanomedicine: polyhedral oligomeric silsesquioxane (POSS). Macromolecular rapid communications. 2011;32:1032-46 16. R. Y. Kannan, H. J. Salacinski, M. Odlyha, P. E. Butler and A. M. Seifalian, The degradative resistance of polyhedral oligomeric silsesquioxane nanocore integrated polyurethanes: an in vitro study. Biomaterials. 2006;27:1971-9

17. G. Punshon, D. S. Vara, K. M. Sales, A. G. Kidane, H. J. Salacinski and A. M. Seifalian, Interactions between endothelial cells and a poly (carbonate-silsesquioxane-bridge-urea) urethane. Biomaterials. 2005;26:6271-9

18. R. Chawla, A. Tan, M. Ahmed, C. Crowley, N. S. Moiemen, Z. Cui, et al., A polyhedral oligomeric silsesquioxane-based bilayered dermal scaffold seeded with adipose tissue-derived stem cells: in vitro assessment of biomechanical properties. journal of surgical research. 2014;188:361-72 19. K. Chaloupka, M. Motwani and A. M. Seifalian, Development of a new lacrimal drainage conduit using POSS nanocomposite. Biotechnology and applied biochemistry. 201 1;58:363-70 20. P. Klanrit, W. Loh, A. Darbyshire, B. G. Cousins, M. Birchall and A. M. Seifalian, Development of 3D tissue engineered larynx scaffold using POSS-nanocomposite materials. Current Opinion in Biotechnology. 2013:S47 
21. M. Baguneid, A. de Mel, L. Yildirimer, B. J. Fuller, G. Hamilton and A. M. Seifalian, In vivo study of a model tissue-engineered small-diameter vascular bypass graft. Biotechnology and applied biochemistry. 2011;58:14-24

22. L. Nayyer, M. Birchall, A. M. Seifalian and G. Jell, Design and development of nanocomposite scaffolds for auricular reconstruction. Nanomedicine: Nanotechnology, Biology and Medicine.

2014;10:235-46

23. I. Ahmed, M. Lewis, I. Olsen and J. Knowles, Phosphate glasses for tissue engineering: part 2. Processing and characterisation of a ternary-based $\mathrm{P} 2 \mathrm{O}$ 5-CaO-Na $2 \mathrm{O}$ glass fibre system. Biomaterials. 2004;25:501-7

24. I. Ahmed, M. Lewis, I. Olsen and J. Knowles, Phosphate glasses for tissue engineering: part 1. Processing and characterisation of a ternary-based $\mathrm{P} 2 \mathrm{O} 5-\mathrm{CaO}-\mathrm{Na} 2 \mathrm{O}$ glass system. Biomaterials. 2004;25:491-9

25. S. N. Nazhat, E. A. Abou Neel, A. Kidane, I. Ahmed, C. Hope, M. Kershaw, et al., Controlled microchannelling in dense collagen scaffolds by soluble phosphate glass fibers. Biomacromolecules. 2007;8:543-51

26. R. Y. Kannan, H. J. Salacinski, P. E. Butler and A. M. Seifalian, Polyhedral oligomeric silsesquioxane nanocomposites: the next generation material for biomedical applications. Accounts of chemical research. 2005;38:879-84

27. S. A. Biela, Y. Su, J. P. Spatz and R. Kemkemer, Different sensitivity of human endothelial cells, smooth muscle cells and fibroblasts to topography in the nano-micro range. Acta Biomaterialia. 2009;5:2460-6

28. A. Kapoor, E. H. Caporali, P. J. Kenis and M. C. Stewart, Microtopographically patterned surfaces promote the alignment of tenocytes and extracellular collagen. Acta biomaterialia. 2010;6:25809

29. E. Martinez, E. Engel, J. A. Planell and J. Samitier, Effects of artificial micro- and nanostructured surfaces on cell behaviour. Ann Anat. 2009;191:126-35

30. R. B. Vernon, M. D. Gooden, S. L. Lara and T. N. Wight, Microgrooved fibrillar collagen membranes as scaffolds for cell support and alignment. Biomaterials. 2005;26:3131-40

31. X. Walboomers, H. Croes, L. Ginsel and J. Jansen, Contact guidance of rat fibroblasts on various implant materials. Journal of biomedical materials research. 1999;47:204-12

32. J. A. Schmidt and A. F. von Recum, Macrophage response to microtextured silicone. Biomaterials. 1992;13:1059-69

33. X. Walboomers, W. Monaghan, A. Curtis and J. Jansen, Attachment of fibroblasts on smooth and microgrooved polystyrene. Journal of Biomedical Materials Research. 2001;55:669-

34. X. Shi, Y. Zhao, J. Zhou, S. Chen and H. Wu, One-step generation of engineered drug-laden poly(lactic-co-glycolic acid) micropatterned with Teflon chips for potential application in tendon restoration. ACS Appl Mater Interfaces. 2013;5:10583-90

35. C. Yang, G. Deng, W. Chen, X. Ye and X. Mo, A novel electrospun-aligned nanoyarnreinforced nanofibrous scaffold for tendon tissue engineering. Colloids Surf B Biointerfaces. 2014;122:270-6

36. Q. Lu, A. Simionescu and N. Vyavahare, Novel capillary channel fiber scaffolds for guided tissue engineering. Acta biomaterialia. 2005;1:607-14

37. G. Wang, S. Moya, Z. Lu, D. Gregurec and H. Zreiqat, Enhancing orthopedic implant bioactivity: refining the nanotopography. Nanomedicine. 2015;10:1327-41

38. C. Vaquette, C. Kahn, C. Frochot, C. Nouvel, J. L. Six, N. De Isla, et al., Aligned poly

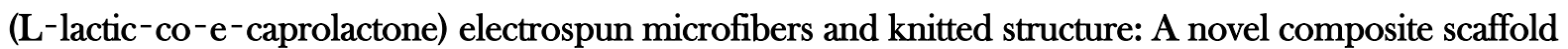
for ligament tissue engineering. Journal of Biomedical Materials Research Part A. 2010;94:1270-82 39. Y. Wan, Y. Wang, Z. Liu, X. Qu, B. Han, J. Bei, et al., Adhesion and proliferation of OCT-1 osteoblast-like cells on micro-and nano-scale topography structured poly (L-lactide). Biomaterials. 2005;26:4453-9

40. C. A. Bashur, R. D. Shaffer, L. A. Dahlgren, S. A. Guelcher and A. S. Goldstein, Effect of fiber diameter and alignment of electrospun polyurethane meshes on mesenchymal progenitor cells. Tissue Engineering Part A. 2009;15:2435-45

41. K. W. Ng, D. T. Leong and D. W. Hutmacher, The challenge to measure cell proliferation in two and three dimensions. Tissue Engineering. 2005;11:182-91 
42. F. Li, B. Li, Q. M. Wang and J. H. C. Wang, Cell shape regulates collagen type I expression in human tendon fibroblasts. Cell motility and the cytoskeleton. 2008;65:332-41

43. S. Sahoo, L.-T. Ang, J. C.-H. Goh and S.-L. Toh, Bioactive nanofibers for fibroblastic differentiation of mesenchymal precursor cells for ligament/tendon tissue engineering applications. Differentiation. 2010;79:102-10

44. T. K. Teh, S.-L. Toh and J. C. Goh, Aligned fibrous scaffolds for enhanced mechanoresponse and tenogenesis of mesenchymal stem cells. Tissue Engineering Part A. 2013;19:1360-72

45. L. Sun, H. Li, L. Qu, R. Zhu, X. Fan, Y. Xue, et al., Immobilized Lentivirus Vector on Chondroitin Sulfate-Hyaluronate Acid-Silk Fibroin Hybrid Scaffold for Tissue-Engineered LigamentBone Junction. BioMed research international. 2014;2014

\section{Figure captions}


Figure 1. Scaffold fabrication process: Glass mold $(16 \times 16 \mathrm{~cm})$ was used as substrate where polymer solution is casted. (A.) Casting a $20 \%$ polymer solution into the mold produces flat scaffold samples. (B.) patterned samples produced by incorporating aligned glass fibers as sacrificial template at the interface of casted polymer, glass fibers were removed by washing samples for $24 \mathrm{hrs}$. In deionized water.

Figure 2. Removal of glass fibers from interface of scaffolds was monitored by measuring the change in dry weight in "grams" (A) and $\mathrm{pH}(\mathbf{B})$ of samples. Error bars are standard error of mean, where $n=4$. SEM images show micro-topography of embedded glass fibers at interphase of the POSS-PCU scaffold (C), and image of scaffold after fibers were removed by dissolution in di-ionized water leaving micro topography at interface of POSS-PCU samples (D). Scale bar represents $200 \mu \mathrm{m}$. Representative cross-section samples are shown at the bottom of each group. Scale bar represents $20 \mu \mathrm{m}$.

Figure 3. Reproducibility of patterned POSS-PCU scaffolds assessed using Light microscopy (A) shows no significant difference in distance between patterns ( $>>0.05)$, Error bars are standard error of mean, where $n=3$. Static water contact angle of different scaffolds is shown (B). Error bars are standard error of mean, where $n=5$. Statistical significance is reported with respect to flat POSS-PCU sample.

Figure 4. Metabolic activity measured by AlamarBlue ${ }^{\circledR}$ assay (A) and cellular growth measured by DNA Assay (B) for cells seeded on both flat and patterned POSS-PCU. Error bars are standard error of mean, where $n=4$. Statistical significance is reported with respect to flat POSS-PCU samples.

Figure 5. Cellular alignment was represented by Actin cytoskeleton staining for cells seeded on flat (A) and pattered POSS-PCU after 24hrs of culture. Reference axis used for calculation of cellular angle for Flat and patterned surface is represented by (a) and (a') respectively. Summary of obtained angulation values represented in (B). Cell attachment after 24 hrs. was calculated by measuring number of DAPI stained nuclei per sample (C) with no significant difference found $(p>0.05)$. Error bars are standard error of mean, where $n=4$. Scale bar represents $200 \mu \mathrm{m}$.

Figure 6. Total collagen production ( $\mathrm{SirCol}^{\mathrm{TM}}$ assay). Functional activity of seeded Tenocytes on different scaffolds was assessed through total collagen production. Error bars are standard error of mean, where $n=4$.

Figure. 7. Immunofluorescence staining for collagen-I (green) and collagen -III (red) expressed by human tenocytes on flat and patterned POSS-PCU samples after 21 days post seeding, where A and D represent flat and B, C, E and F represent patterned POSS-PCU samples. $\mathrm{G}$ and $\mathrm{H}$ represent negative control for collagen -III and collagen -I respectively. Due to excessive background during imaging, images on patterned samples were presented separately for collagen -I/ collagen -III and DAPI staining. Scale bar represents $200 \mu \mathrm{m}$.

Figure. 8. Immunofluorescence staining for tenascin-C (green) and scleraxis (red) expressed by human tenocytes on flat and patterned POSS-PCU samples after 21 days post seeding, where A and D represent flat and B, C, E and F represent patterned POSS-PCU samples. G and $\mathrm{H}$ represent negative control for scleraxis and tenascin- $\mathrm{C}$ respectively. Due to excessive background during imaging, images on patterned samples were presented separately for tenascin-C/scleraxis and DAPI staining. Scale bar represents $200 \mu \mathrm{m}$. 


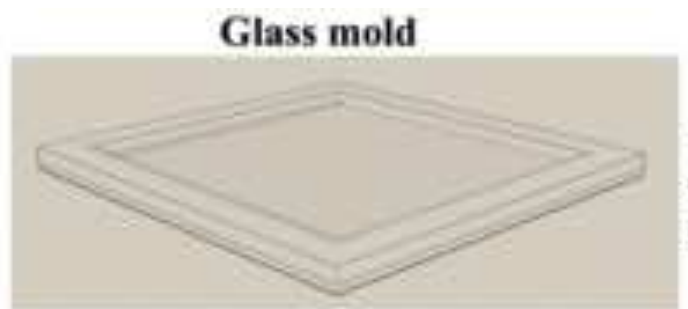

$16 \times 16 \mathrm{~cm} \mathrm{glass}$ container was used as a casting mold
A. Production of flat polymer films
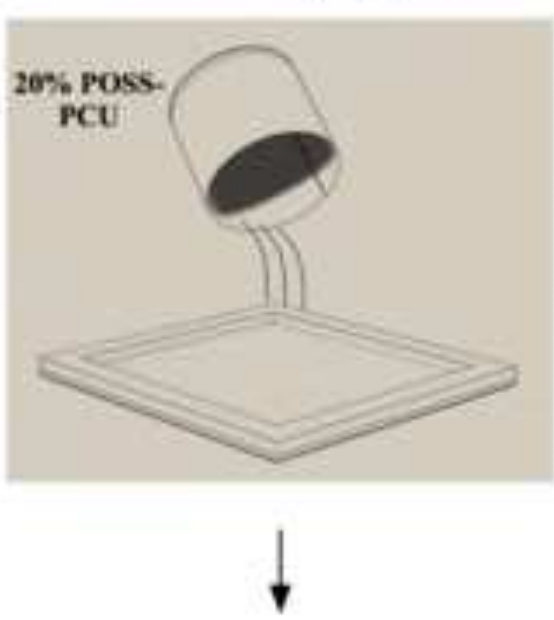

After 16 hours of solvent evaporation

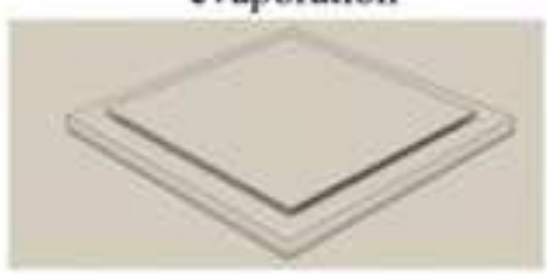

Flat film is produced
B. Production of patterned polymer

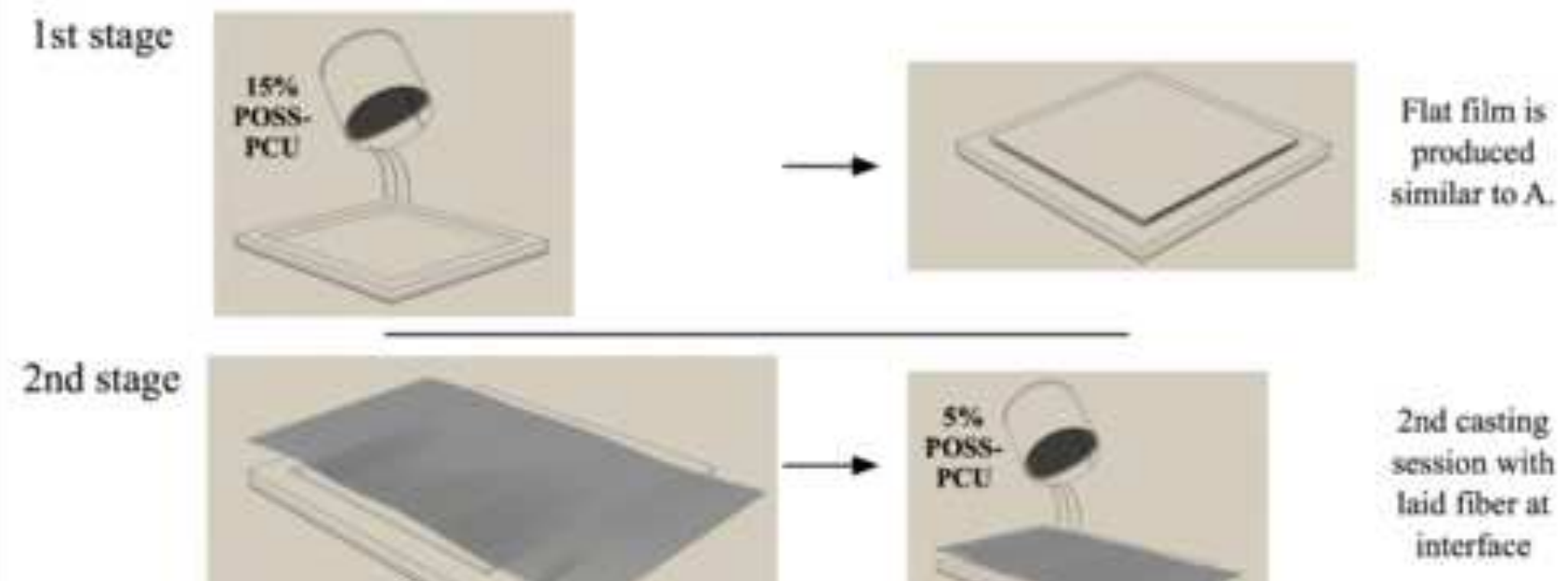

Final product

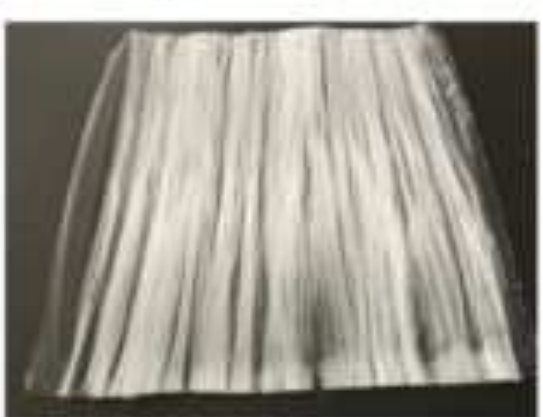

Polymer film after two stages of casting

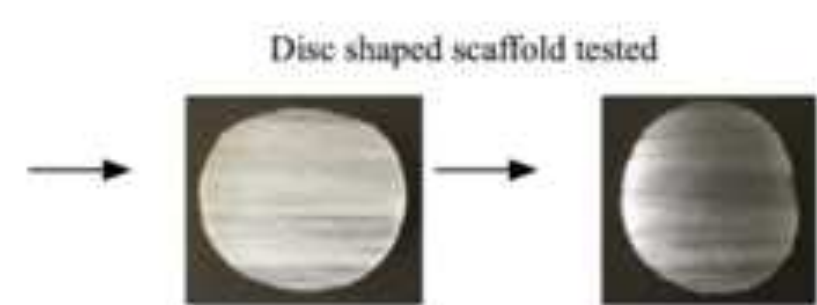

Samples prior to glass Samples after dilution of fiber dilution (Patterned-POSS-PCU) 
Click here to download high resolution image
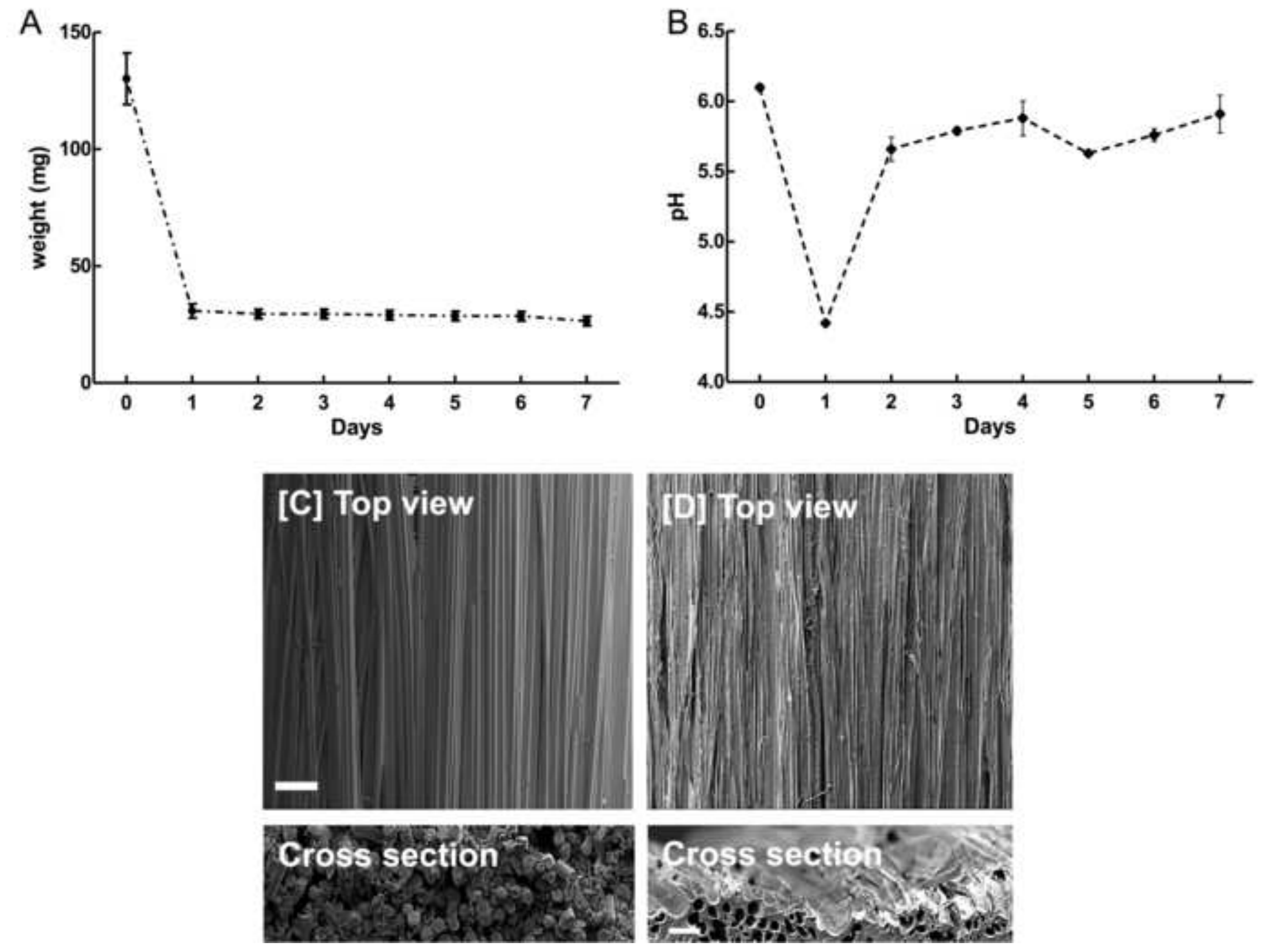
Click here to download high resolution image

A

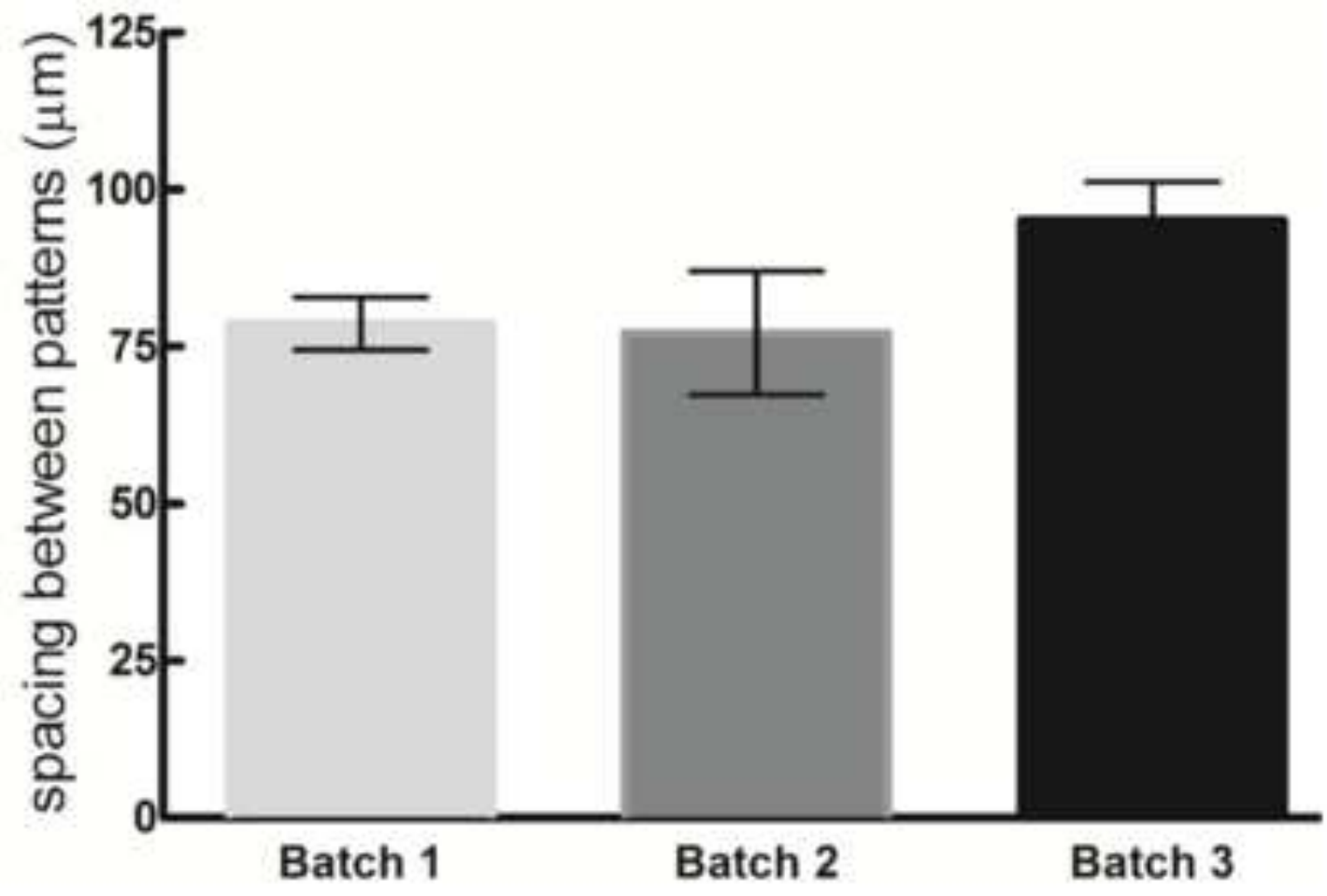

B

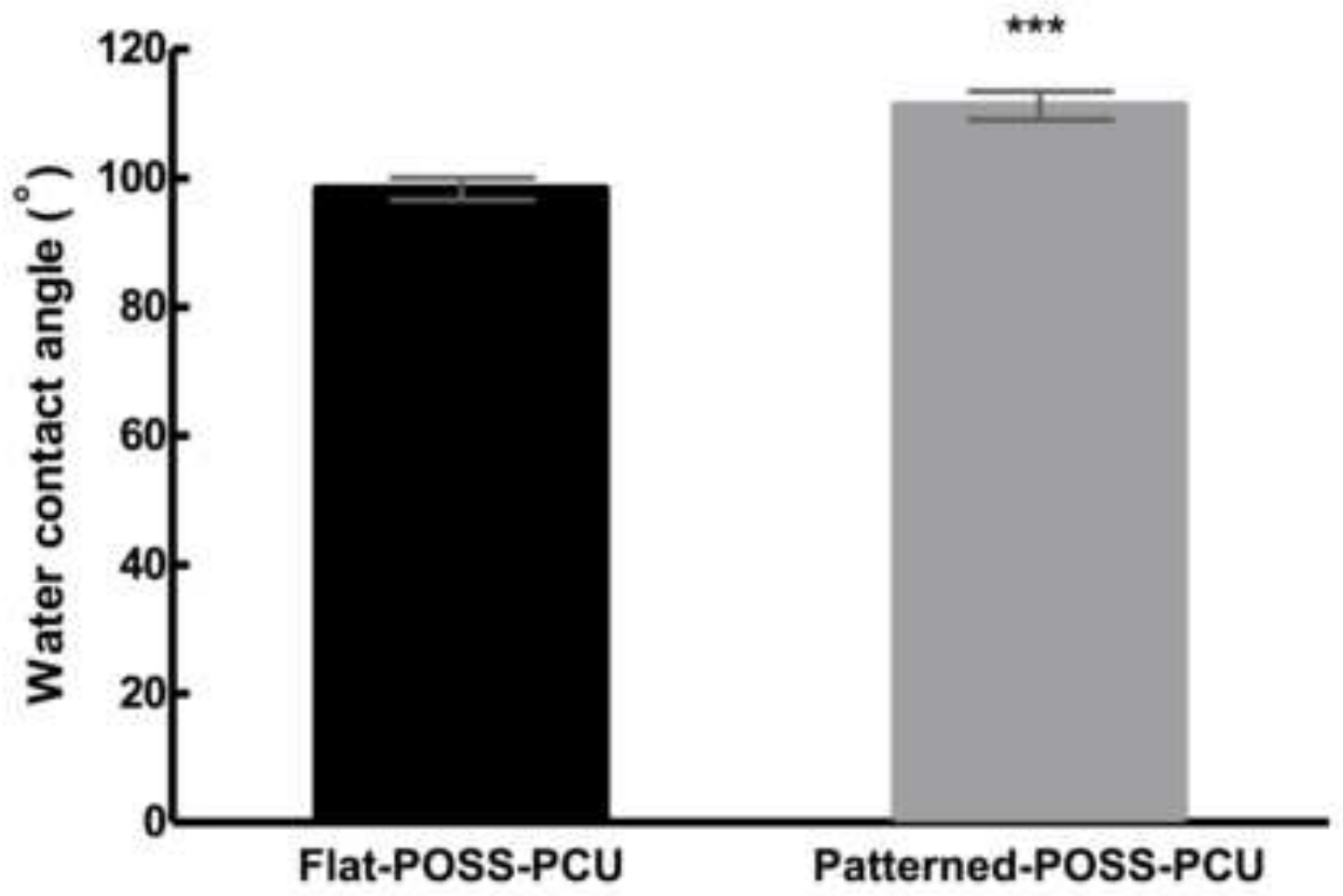


Figure 4
Click here to download high resolution image

A

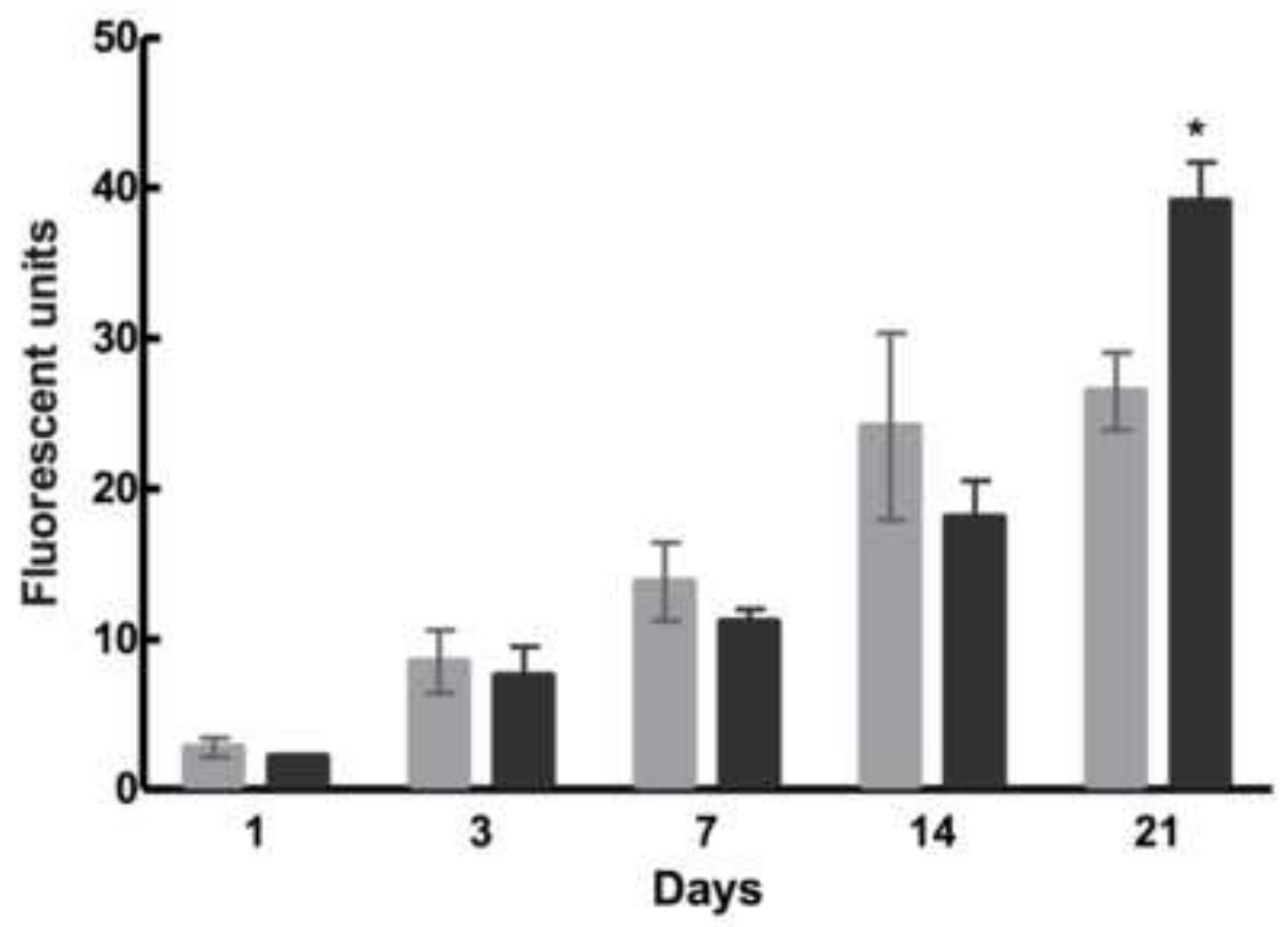

B

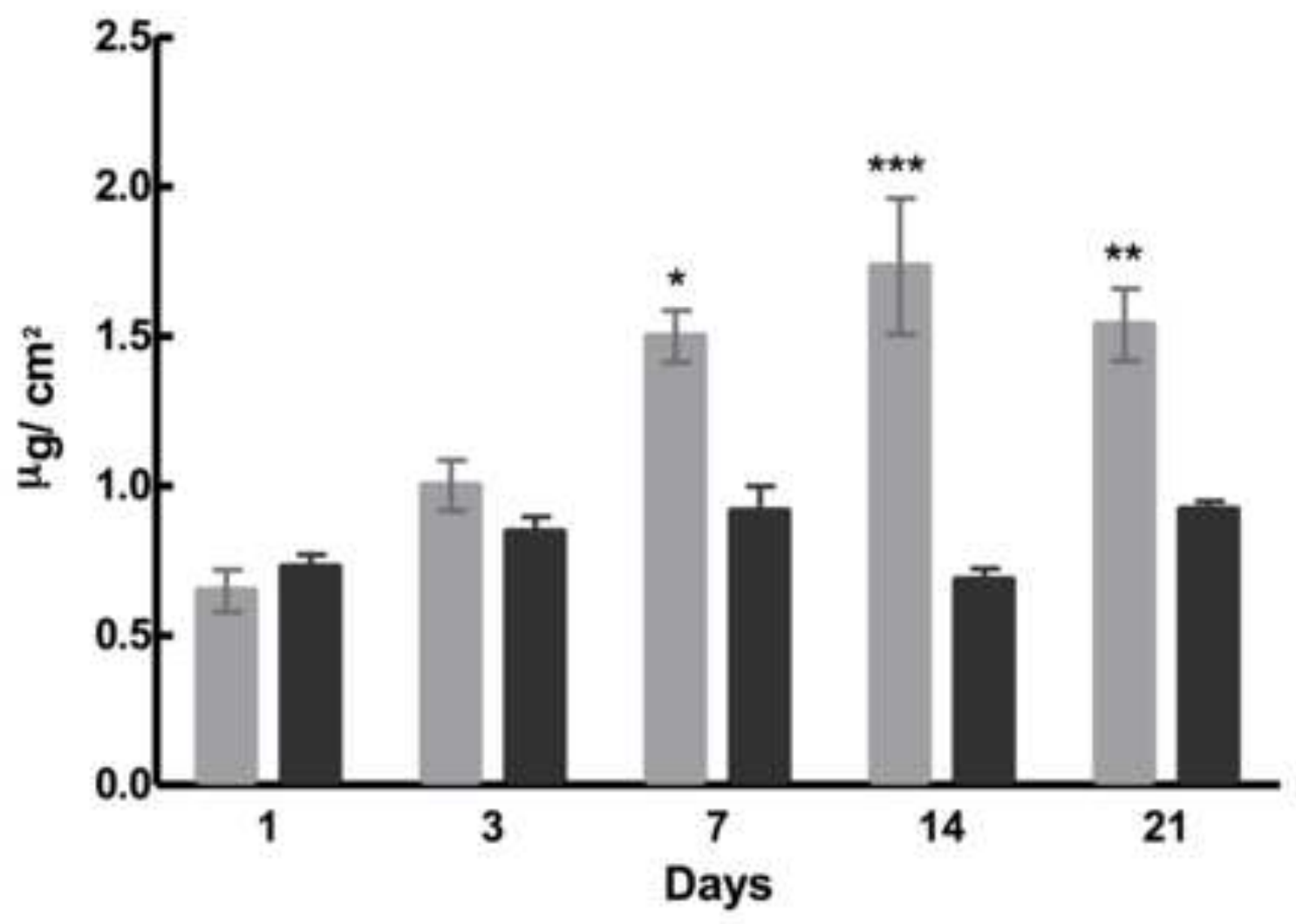


Click here to download high resolution image
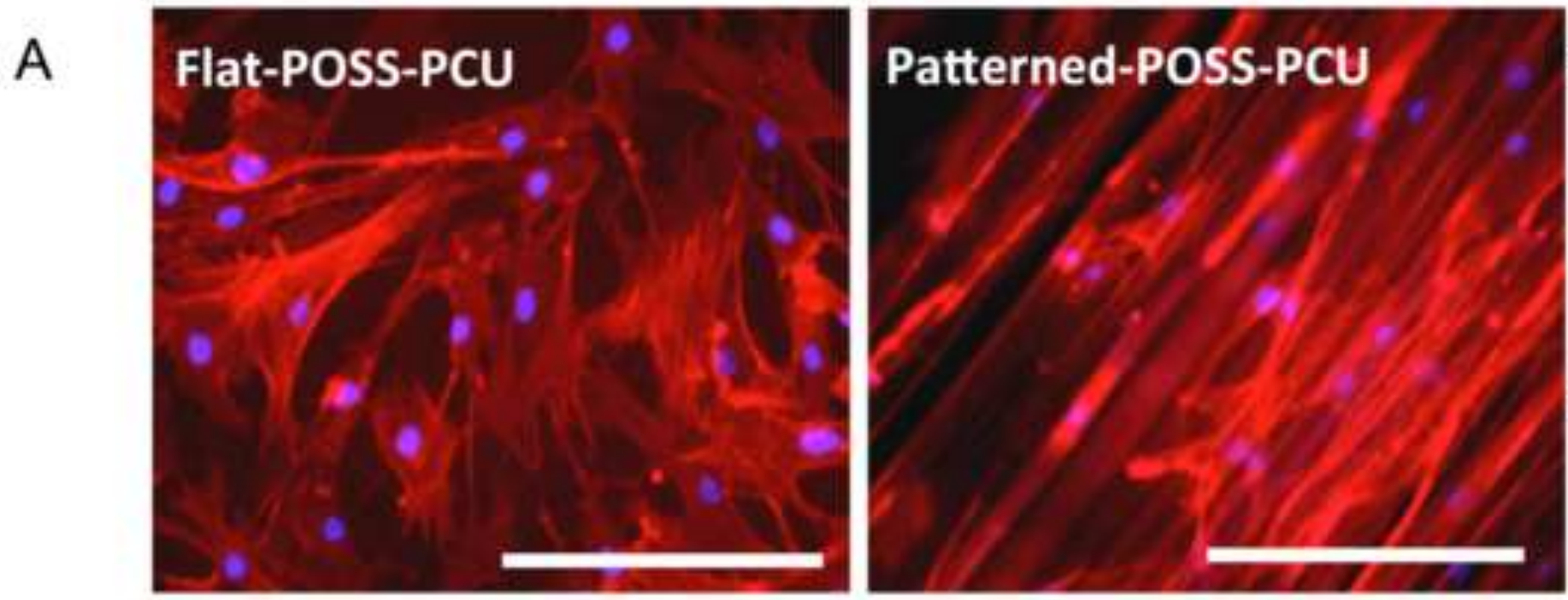

a.

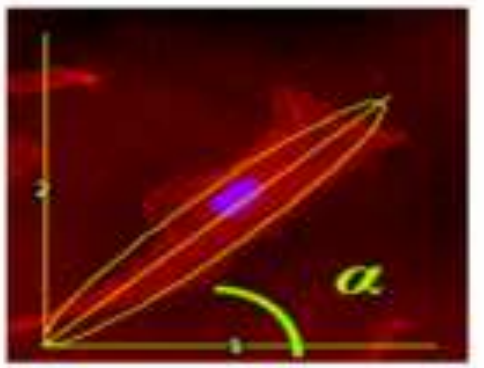

$a^{\prime}$.

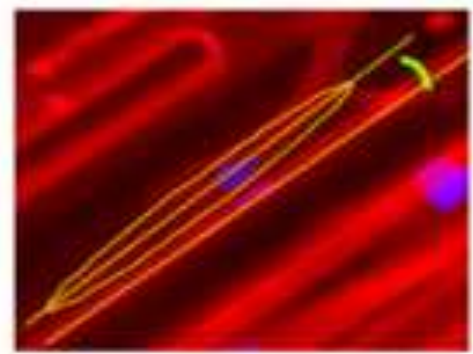

B

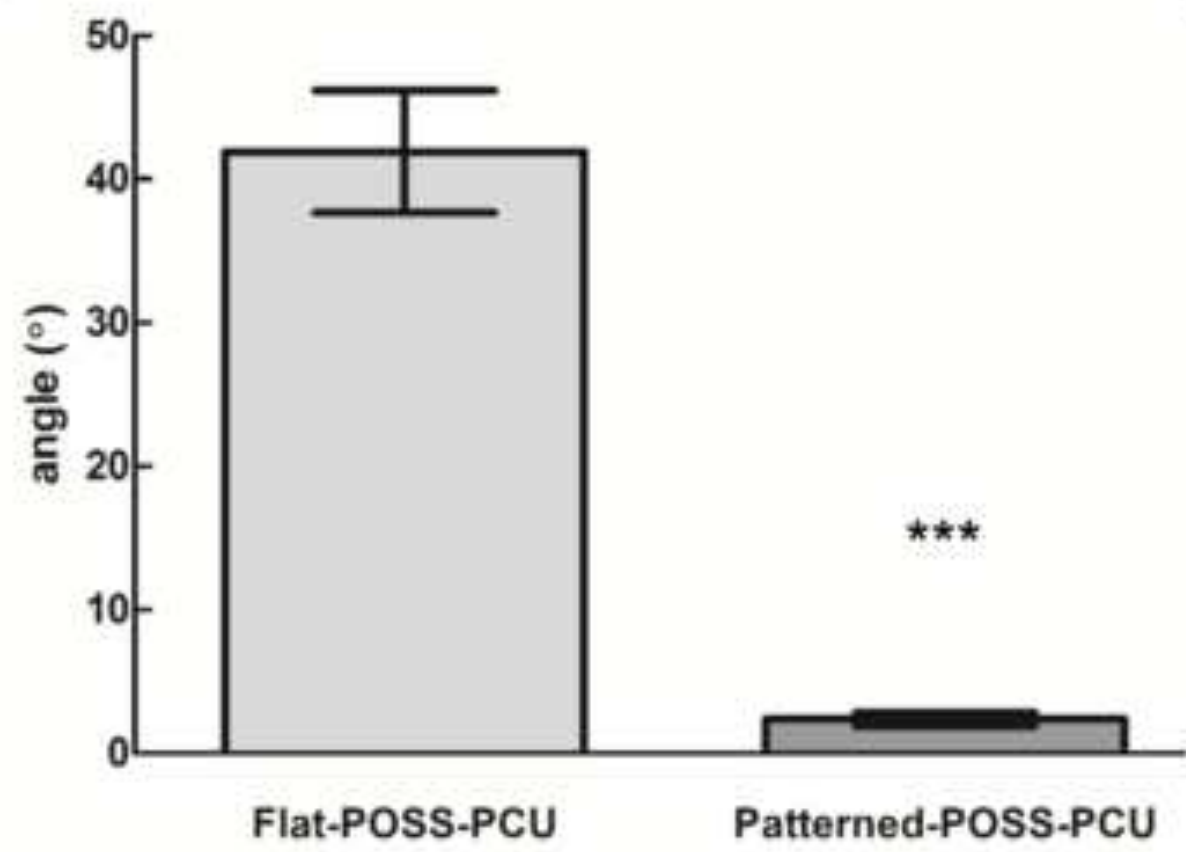

C

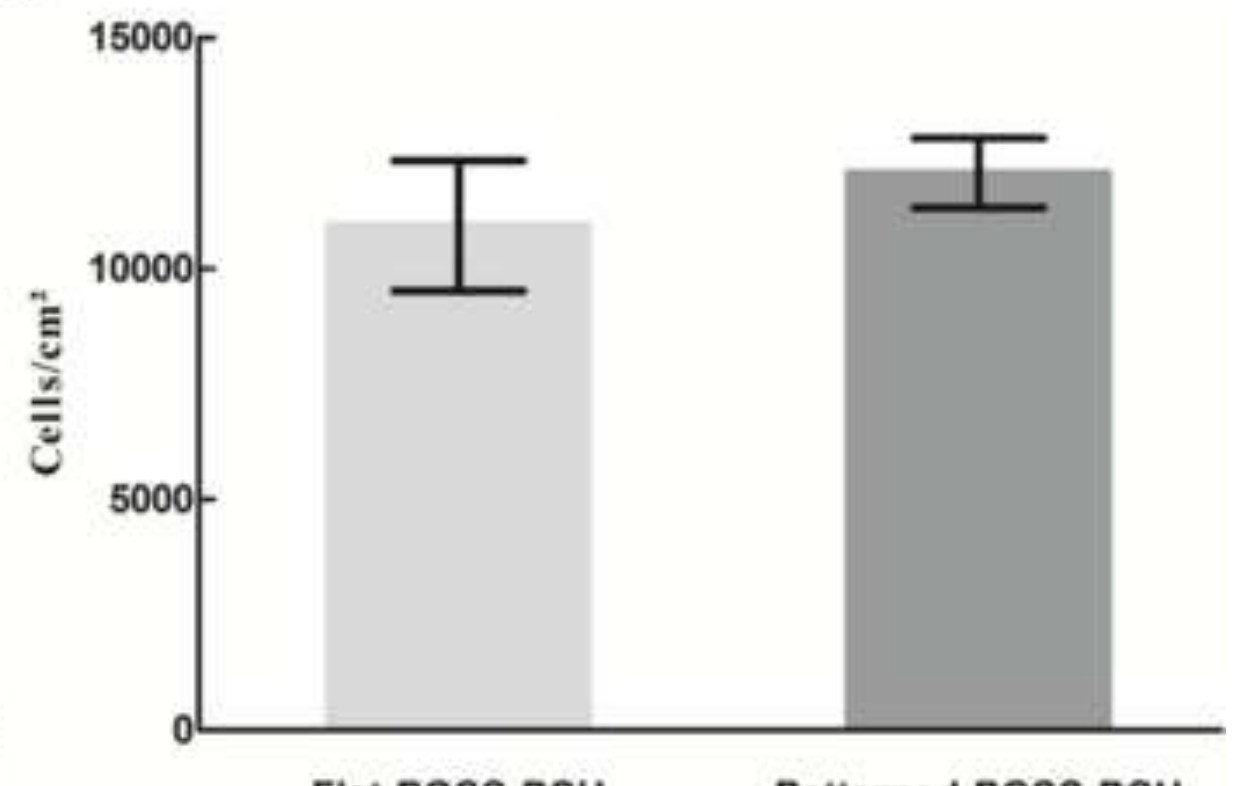

Flat-POSS-PCU

Patterned-POSS-PCU 


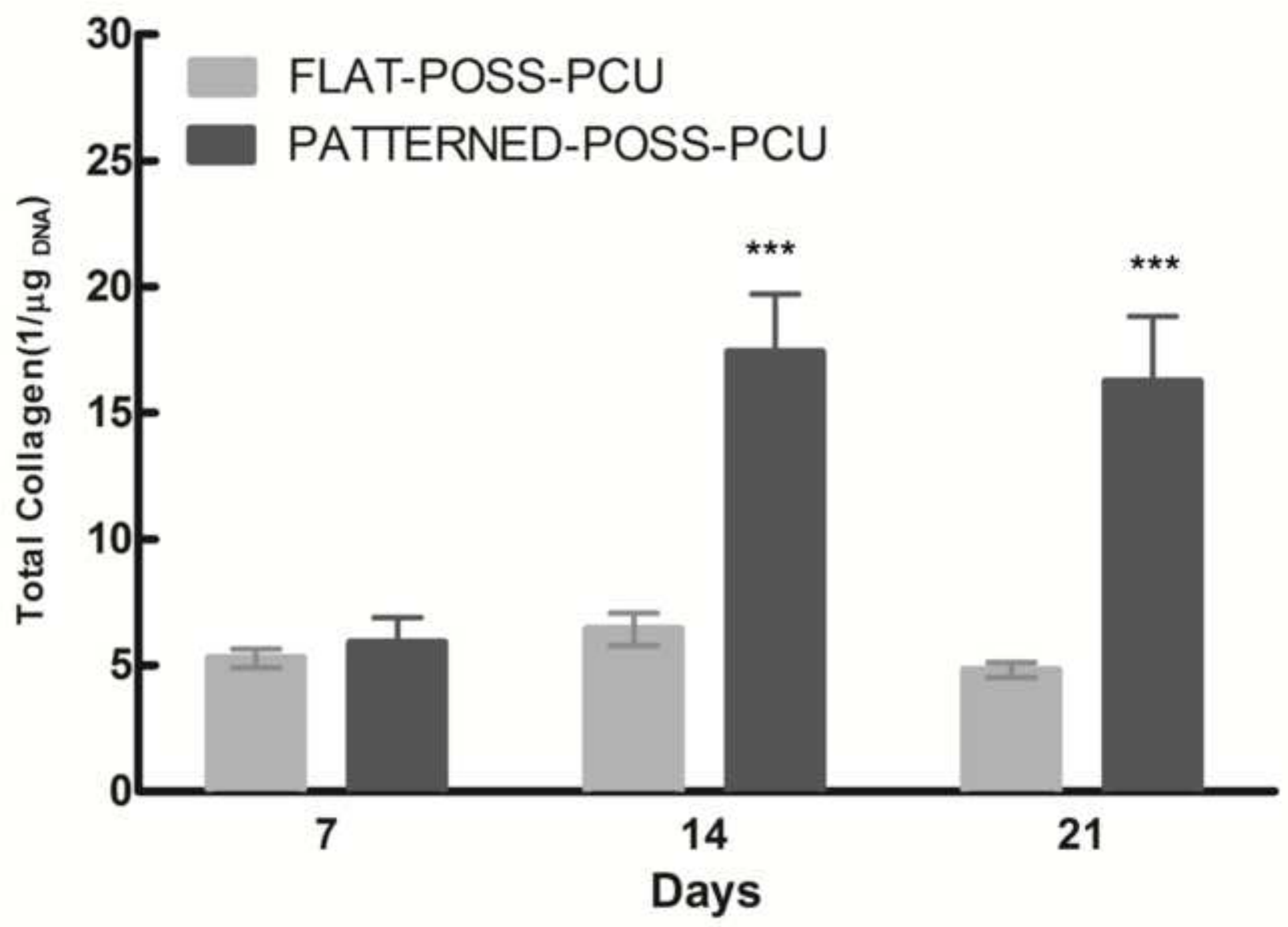



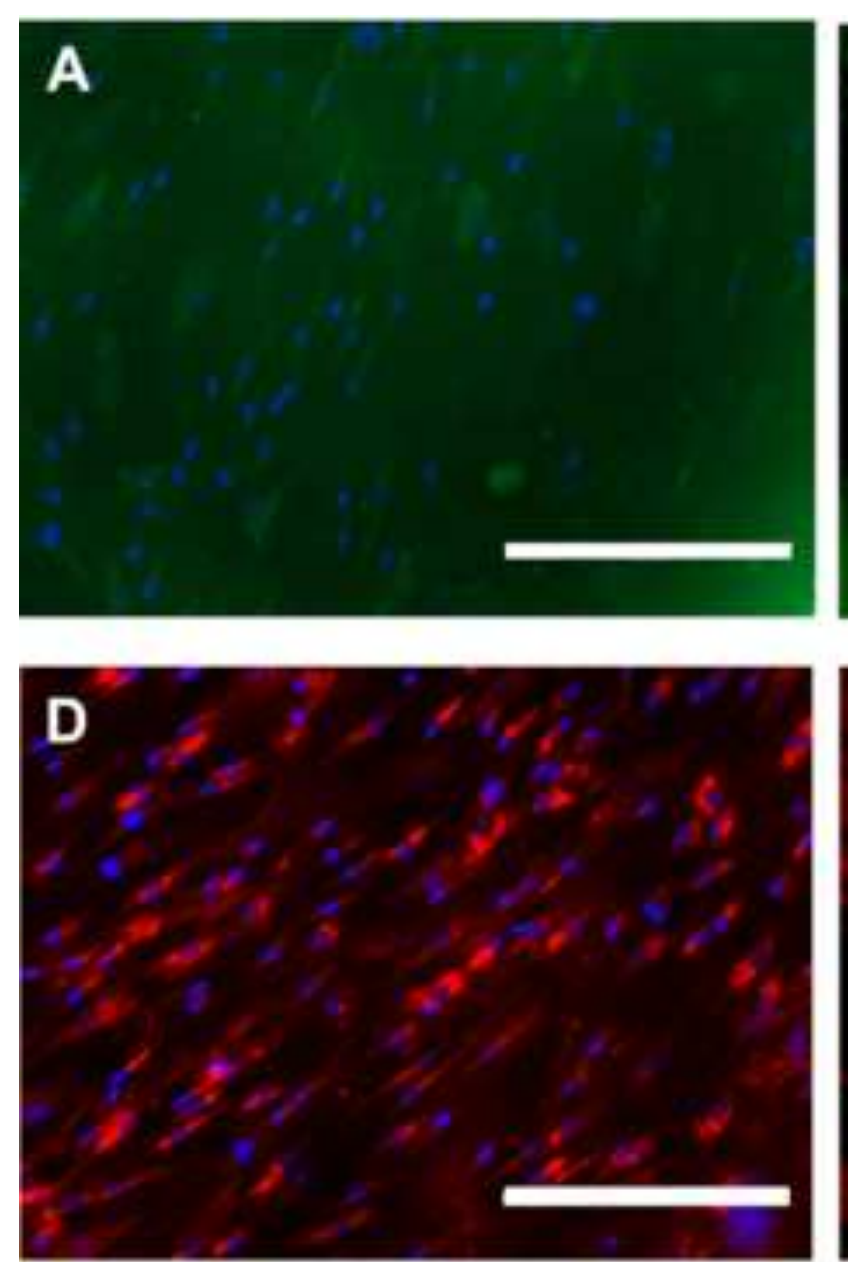

E

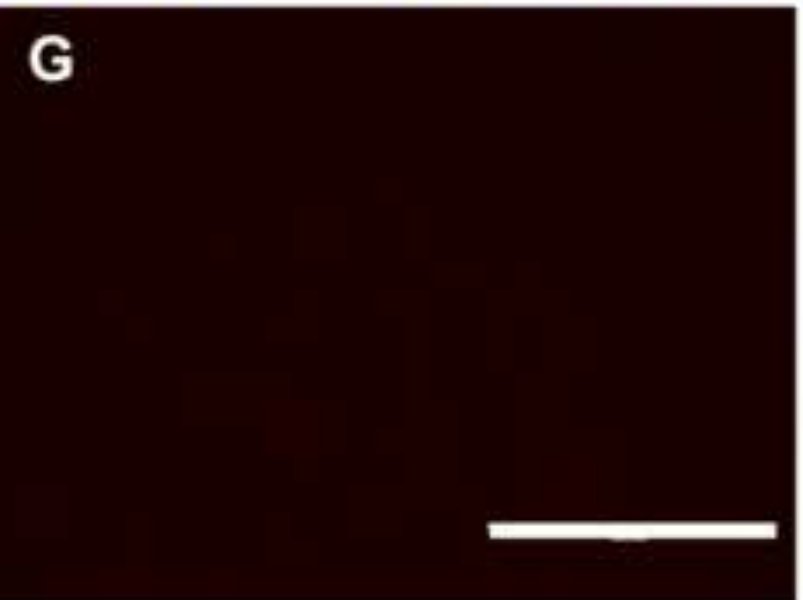

H

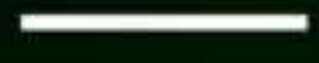


Click here to download high resolution image
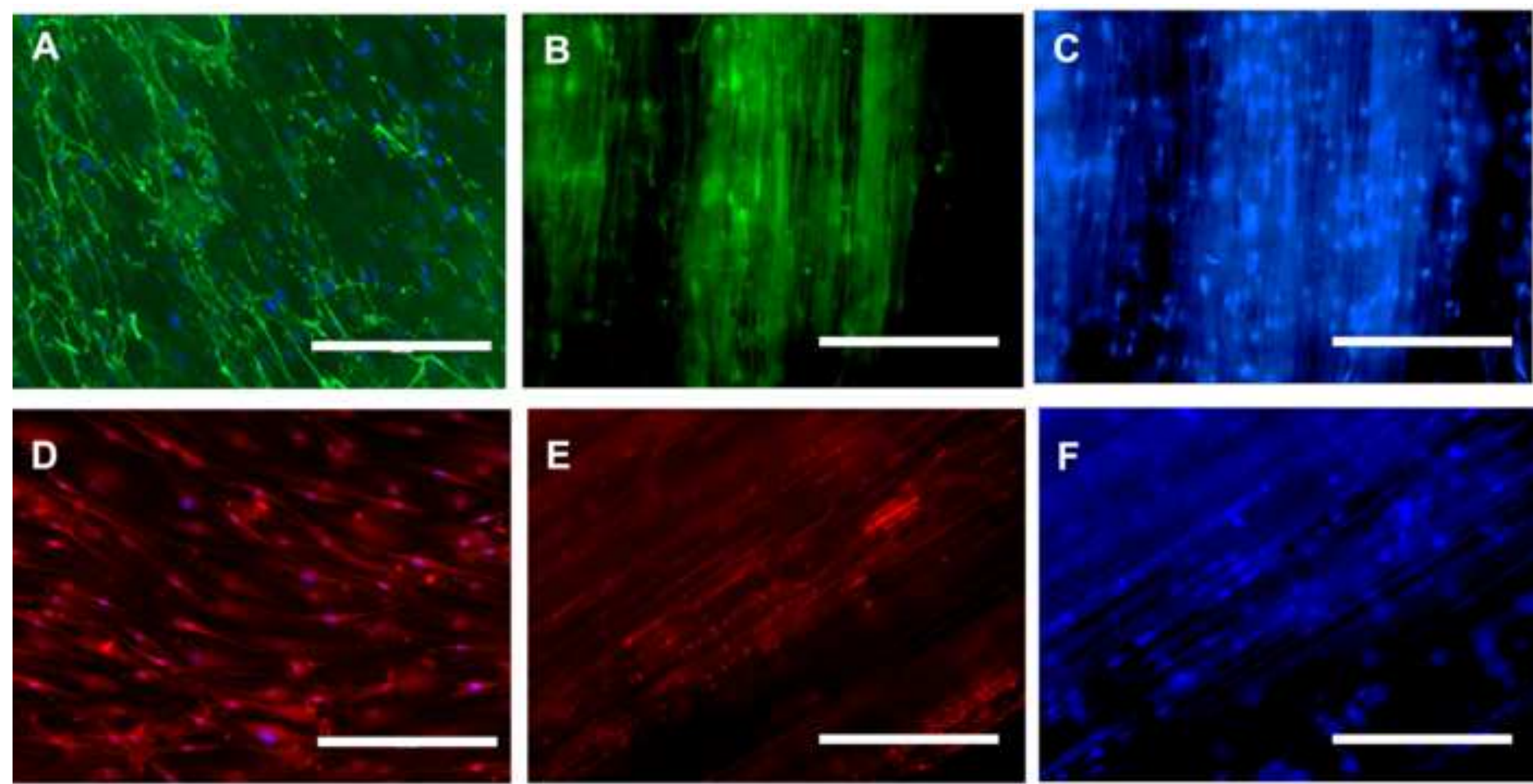

E

$\mathbf{F}$

G

H
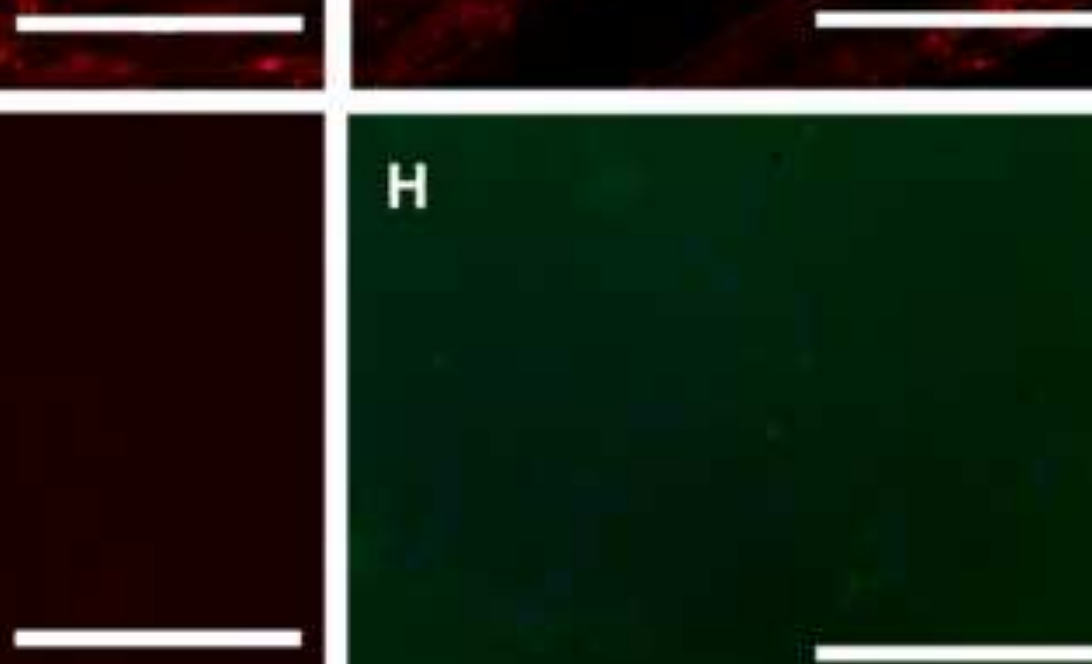
Supplementary Material
Click here to download Supplementary Material: Supplimentry information Nanomedicine.pdf

Supplementary Material
Click here to download Supplementary Material: Supplimentry information Nanomedicine.pdf

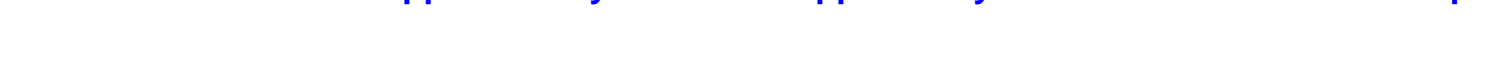

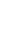

df

(1)

$\sqrt{3}$




\section{NANOMEDICINE: NBM}

hltp://www.nanomedjournal.com/

htt p://ww w.ees.elsc vier.com/nano/

nnbu.journalofrice(a) guail.com

\section{AUTHORSHIP AGREEMENT \\ Must be signed by ALL authors \\ Scan and upload with your submission.}

In accordance with the Authorship and Contributorship policy available at www.icmje.org, the undersigned certify that each participant: 1) made substantial contributions to conception and design, or acquisition of data, or analysis and interpretation of data; 2) drafted the article or revised it critically for important intellectual content; and 3) gives final approval of the submitted manuscript, revised versions, and version to be published. A uthors should meet conditions 1, 2, and 3.

Manuscript \#_JN2016319R1

Title: Micro Patterning of nanocomposite polymer Scaffolds Using Sacrificial Phosphate Glass Fibres for Tendon Tissue Engineering Applications

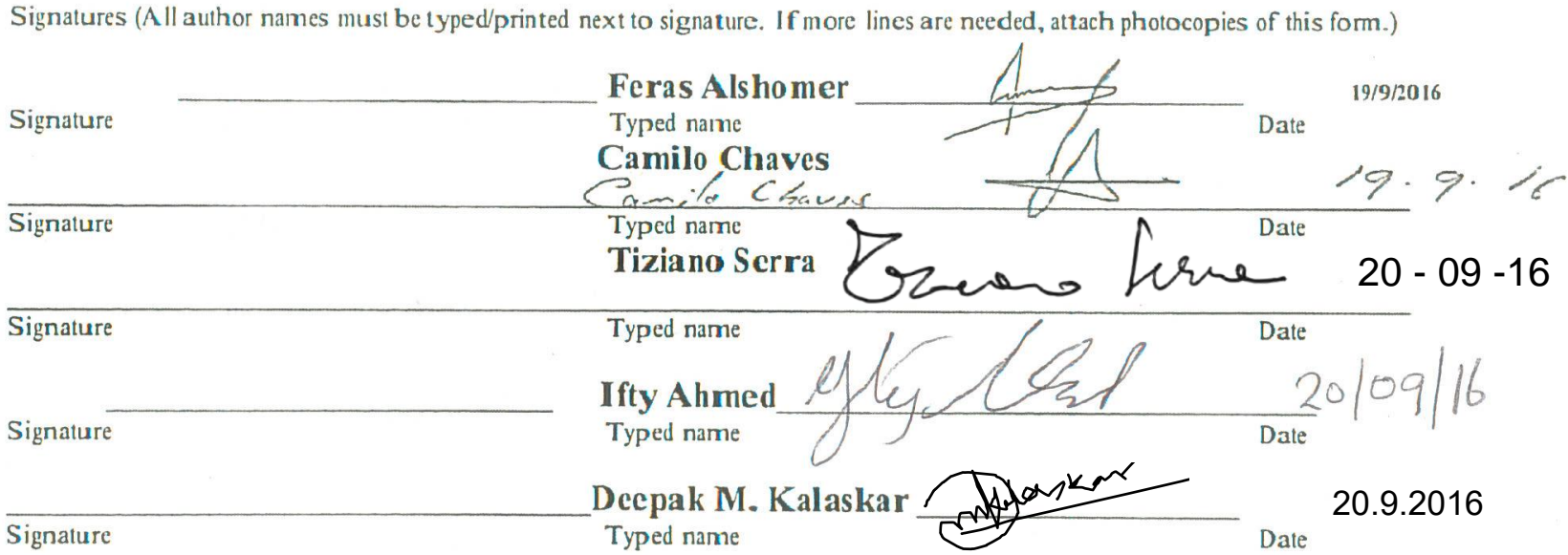

